\title{
Why are macroscopic experiments reproducible? Imitating the behavior of an ensemble by single pure states
}

\author{
Peter Reimann* \\ Fakultät für Physik, Universität Bielefeld, 33615 Bielefeld, Germany \\ Jochen Gemmen \\ Department of Physics, University of Osnabrück, D-49069 Osnabrück, Germany
}

\begin{abstract}
Evidently, physical experiments are practically reproducible even though the fully identical preparation of initial state wave functions is often far beyond experimental possibilities. It is thus natural to explore if and in which sense specific, uncontrollable features of initial wave functions are irrelevant for the observable course of an experiment. To this end we define ensembles of pure states which are then shown to generate extremely similar non-equilibrium dynamics of the expectation values of practically all standard observables. The ensembles are constructed to comply with some reduced, coarse a priori information on the state of the system, like, e.g. a few specific expectation values, etc. However, different types of ensembles with different additional properties are possible. We discuss some of them.
\end{abstract}

\section{INTRODUCTION}

Appreciating simplicity and surprises on a fundamental level were among the main things we could learn from our unforgettable friend and teacher Chris Van den Broeck. One of us (P.R.) had the great privilege to spend more than two years with him as a postdoc during the heyday of exploring neural networks in terms of statistical mechanics. In this context, he happily infected all of us with his excitement about the intriguing geometrical properties of high-dimensional Euclidean spaces [1], such as the well-known peculiarities of a simple sphere: Almost its entire volume is concentrated in an exceedingly thin surface layer, which, in turn, exhibits an extreme concentration around a very narrow "equatorial belt" or any other "great circle", and hence also around the intersection of two such circles, and so on.

Some ten years later, a related surprise in highdimensional Hilbert spaces attracted a lot of interest under the labels of "canonical typicality" 2] and "concentration of measure" [3]. Namely, the rediscovery [4] that the overwhelming majority of randomly sampled wave functions (vectors on the unit sphere) exhibit almost identical expectation values. Obviously, this was exactly the type of thing which fascinated Chris, in particular since he immediately recognized its far reaching physical implications: All thermal fluctuation effects could, in principle, be ascribed to the purely quantum mechanical fluctuations of a pure state [4]. Yet he remained unhappy with the rather unintuitive "Levy lemma", exploited in [3] to derive those results, but only for a few days; the following email from March 2007, entitled "Levy lemma obvious", reminds us once more of his inimitable style: "Yes it is obvious, I have understood it while sitting in the

\footnotetext{
*Electronic address: reimann@physik.uni-bielefeld.de
}

${ }^{\dagger}$ Electronic address: jgemmer@uos.de bath, like the great Archimedes ... Consider the (closed) line of points $x$ on the sphere for which $f(x)$ takes on the median value. This line divides by definition the sphere in two equal parts. This line is at least in length like an equator. Any small neighborhood of the equator basically covers the whole sphere in high dimensions. This is then a fortiori so for the neighborhood of the median line, which is due to the Lipschitz condition also the values of $f$ close to the median. That is it."

At the focus of our present work is the question of how far one can go in extending those originally nondynamical typicality concepts [2 4 into the realm of dynamical typicality [5], and how they can be efficiently utilized for practical (analytical and numerical) purposes.

\section{MAIN ISSUES AND RESULTS}

\section{A. Core message}

It is a basic scientific principle and empirical fact that repeating a macroscopic experiment at different times or in different labs results in reproducible observations. On the other hand, some small differences in the microscopic initial conditions are practically unavoidable and will subsequently grow very fast due to the generically chaotic dynamics, so that the similarity of the final results becomes difficult to explain on a fundamental theoretical level.

Leaving aside the technical details, the main result of our present explorations provides the following general picture of how the above antinomy can be resolved within the framework of elementary quantum mechanics: Taking for granted that the initial system energy is known (is reproducible) up to an uncertainty which is small on macroscopic but large on microscopic scales, the overwhelming majority of initial (pure) states $|\psi\rangle$ which are compatible with this information (energy) yields very similar expectation values $\langle\psi|O| \psi\rangle$ for any given observ- 
able $O$, which in turn is very close to the expectation value $\operatorname{Tr}\left\{\rho_{\mathrm{mc}} O\right\}$, where $\rho_{\mathrm{mc}}$ is the textbook microcanonical ensemble for the given system energy. In fact, this is the essence of the well-known (canonical) typicality and concentration of measure phenomena [2 4, 6 10], which were mentioned already in Sec. I

To characterize a non-equilibrium initial condition, additional information (besides the system energy) is thus indispensable. In the simplest case, it is known that the expectation value $\left\langle\psi\left|A_{1}\right| \psi\right\rangle$ of some observable $A_{1}$ (e.g. some macroscopic system property) notably deviates from the corresponding equilibrium value $\operatorname{Tr}\left\{\rho_{\mathrm{mc}} A_{1}\right\}$, and instead is (approximately) equal to some other value $\alpha_{1}$. Similarly as before, we will show that the vast majority of initial states $|\psi\rangle$ which (approximately) reproduce the given system energy and the non-equilibrium expectation value of $A_{1}$, yield very similar expectation values $\langle\psi|O| \psi\rangle$ also for any other observable $O$. If there is yet another observable $A_{2}$, whose expectation value $\alpha_{2}$ is known to notably deviate from the latter typical value, then there will be yet another ensemble of $|\psi\rangle$ 's, which is compatible with the energy and the given $\alpha_{1}$ and $\alpha_{2}$ values, and which again yields predominantly very similar $\langle\psi|O| \psi\rangle$ values, and so on.

In many experimental situations it seems reasonable to expect that there exists a finite, relatively small number $K$ of such steps, after which all relevant non-equilibrium information will have been satisfactorily taken into account. (Whether or not one explicitly knows all those $A_{k}$ 's and $\alpha_{k}$ 's does not matter.) Furthermore, one expects that the "true" initial state $|\psi\rangle$ now belongs to the concomitant vast majority of states, which all exhibit very similar $\langle\psi|O| \psi\rangle$ values for $O=A_{k}, k=1, \ldots, K$, but also for practically any other physically relevant observable $O$.

By employing the Heisenberg picture of quantum mechanics, one can conclude [7] that also the expectation value of $O$ at any later instant of time will be very similar for most of those initial states $|\psi\rangle$, a property for which the term dynamical typicality has been coined in Ref. 5].

In other words, we propose a new way of classifying non-equilibrium initial states of many-body systems (at a given energy), namely according to the pertinent set of observables $A_{k}$ and their expectation values $\alpha_{k}$. Exactly as in an experiment, for the vast majority of initial states which are compatible with this information, all measurable properties of the system both at the initial and also at any later time point turn out to be very similar (the differences are either below any reasonable resolution limit or their probability is negligibly small). All the further details, which inevitably must remain unknown and irreproducible in an experiment, are thus indeed "irrelevant" in our theory as well.

\section{B. Ramifications}

If the considered system is isolated from the rest of the world, then it will usually exhibit a temporal relaxation towards thermal equilibrium. But it should be emphasized that also systems which do not thermalize and even non-autonomous systems with explicitly time-dependent Hamiltonians are still admitted by the general framework of our present work.

On the other hand, standard quantum mechanics cannot directly deal with open systems, interacting and entangled with some environment. In such a case, we therefore tacitly consider, as usual, the entire system-plusenvironment compound as the actual (super-)system of interest, which then can again be treated as isolated from the rest of the world.

So far, the entire discussion was conducted in terms of expectation values, i.e., ensemble averaged measurement outcomes when repeating the same experiment many times. On the other hand, due to the fundamentally probabilistic nature of quantum mechanics, even when the considered setup (system state and observable) is always exactly identical (which is not feasible in a real experiment but is an admissible Gedankenexperiment), the actual outcomes of the single measurements in general give rise to a non-trivial statistical distribution. This issue is of particular relevance for microscopic observables (e.g., the Brownian motion of a small particle, or the velocity distribution of one molecule), where often not only the average but rather the entire measurement outcome distribution may be of interest. The corresponding generalization of our approach is straightforward, predicting that even the entire statistics of the different possible measurement outcomes will be almost the same for practically all initial states with common $\left\langle\psi\left|A_{k}\right| \psi\right\rangle$ values. The reason is that with any given observable $O$, also the higher moments $O^{2}, O^{3}, \ldots$, or equivalently, the projectors onto any eigenspace of $O$, are admissible observables with very similar expectation values for most $|\psi\rangle$ 's.

Note that in reality, the actually measurable fluctuations of a microscopic observable (e.g. the position of a fluorescent molecule in a fluid) are unavoidably "contaminated" with differences in the initial states when repeating the "same" experiment. Similarly as in the original (canonical) typicality investigations [2 -4, 6- 10], a conceptual key aspect of our approach is that those microscopic fluctuations would actually remain largely the same even if the initial states were strictly identical in every repetition of the experiment.

In the same vein, our approach allows one to (approximately) preset not only the initial expectation values of certain observables $A_{k}$ but even the entire statistics of their different possible measurement outcomes. For instance, one may impose some expectation value $\alpha_{1}$ for an observable $A_{1}$ and then choose as a second observable $A_{2}:=\left(A_{1}-\alpha_{1}\right)^{2}$ together with a possibly quite small (positive) value of $\alpha_{2}$. In this way, not only the mean value of the (random) measurement outcomes of $A_{1}$, but 
also the variance can be prescribed, and hence will be closely reproduced with high probability by an arbitrary but fixed sample of the corresponding ensemble of pure states $|\psi\rangle$. Analogously, further details of the measurement outcome statistics can be imposed via the third and higher moments of $A_{1}$, or equivalently, via the projectors onto the eigenspaces of $A_{1}$ and the corresponding projection probabilities [11].

Besides the so far discussed lack of knowledge about the exact system state $|\psi\rangle$, also the Hamiltonian, which governs the time-evolution and even the pertinent Hilbert space may not be exactly known and/or may vary upon repeating the "same" experiment. However, this issue goes beyond the scope of our present work.

\section{DYNAMICAL TYPICALITY}

We consider a Hilbert space $\mathcal{H}$, spanned by some orthonormal basis $\left\{\left|\chi_{n}\right\rangle\right\}_{n=1}^{N}$, whose dimension $N$ may be either large but finite or infinite. For instance, every $\left|\chi_{n}\right\rangle$ may be an eigenvector of some Hamiltonian $H$, and $\mathcal{H}$ may be either an energy shell (spanned by a finite subset of all eigenvectors of $H$ ) or the full Hilbert space (spanned by all eigenvectors of $H$ ), see also Sec. VI below.

As in Sec. II we mainly have in mind macroscopic (or "many-body") systems, for which the dimension of a typical microcanonical energy shell is well-know to be exponentially large in the number of microscopic degrees of freedom, while the full Hilbert space will be of even larger and possibly infinite dimensionality.

In terms of the above introduced basis $\left\{\left|\chi_{n}\right\rangle\right\}_{n=1}^{N}$ of $\mathcal{H}$, we define an ensemble of random vectors $|\phi\rangle \in \mathcal{H}$ via

$$
|\phi\rangle=\sum_{n=1}^{N} R c_{n}\left|\chi_{n}\right\rangle
$$

where the $c_{n}$ are complex numbers, whose real and imaginary parts are given by independent, Gaussian distributed random variables of mean zero and variance $1 / 2$. Moreover, $R$ is a linear operator on $\mathcal{H}$, which for the moment may still be arbitrary apart from the requirement that the positive semidefinite Hermitian operator

$$
\rho:=R R^{\dagger}
$$

is of finite trace. Obviously, the latter requirement is non-trivial only if $N$ is infinite and guarantees, as we will see in a moment, that the random vectors in (11) are of finite norm (with probability one). For the rest, the random vectors (1) are not yet normalized, rather their norm is itself a random variable. Moreover, it will turn out that the finite trace of $\rho$ can be set to unity without any loss of generality later on, i.e., we require that

$$
\operatorname{Tr}\{\rho\}=1 .
$$

As anticipated by the notation, $\rho$ is thus a well defined density operator (Hermitian, positive semidefinite, and of unit trace).
Note that $R$ on the right hand side of (1) could be readily taken out of the sum if $N$ is finite, but for infinite $N$ the remaining sum (without $R$ ) would not be well defined.

A key property of the random vector ensemble in (1) is its invariance under arbitrary unitary transformations of the basis $\left|\chi_{n}\right\rangle$ of $\mathcal{H}$ (all statistical properties remain unchanged). In other words, the basis can be chosen arbitrarily. This is of particular interest when numerically sampling random vectors according to (11) on the computer: For instance, any single-particle product basis will do in the case of a many-body system. Furthermore, focusing on the special choice $R=\mathbb{1} / \sqrt{N}$ (which is only possible for $N<\infty$, and where $\mathbb{1}$ is the identity on $\mathcal{H}$ ) we can conclude that after normalization of each random vector in (1), all those normalized vectors will be equally likely (uniformly distributed) in a very natural sense.

Next we consider an arbitrary Hermitian operator $B: \mathcal{H} \rightarrow \mathcal{H}$. If the dimension $N$ of $\mathcal{H}$ is infinite, the spectrum of $B$ is furthermore required to be bounded. In other words, the operator norm $\|B\|$ (largest eigenvalue in modulus) is required to be finite. Since only $B$ 's which model some observable will actually be of interest later on, and since the measurement range of any real observable is finite, the latter requirement does not amount to any significant loss of generality.

Denoting the average over the random vector ensemble from (1) by an overbar, one can readily show that

$$
\begin{aligned}
\bar{B} & :=\overline{\langle\phi|B| \phi\rangle}=\operatorname{Tr}\{\rho B\} \\
\sigma_{B}^{2} & :=\overline{(\langle\phi|B| \phi\rangle-\bar{B})^{2}}=\operatorname{Tr}\left\{(\rho B)^{2}\right\}
\end{aligned}
$$

by means of the following line of reasoning: In order to verify (4), we utilize (1) and the abbreviation $B^{\prime}:=$ $R^{\dagger} B R$ to obtain

$$
\bar{B}=\sum_{m, n=1}^{N} \overline{c_{m}^{*} c_{n}}\left\langle\chi_{m}\left|B^{\prime}\right| \chi_{n}\right\rangle .
$$

Since the real and imaginary parts of the $c_{n}$ 's are independent, Gaussian distributed random variables of mean zero and variance $1 / 2$ (see below (10), it follows that $\overline{c_{m}^{*} c_{n}}=\delta_{m n}$. Eq. (6) thus takes the form

$$
\bar{B}=\sum_{n=1}^{N}\left\langle\chi_{n}\left|B^{\prime}\right| \chi_{n}\right\rangle=\operatorname{Tr}\left\{B^{\prime}\right\}
$$

Exploiting the definition of $B^{\prime}$, the cyclic invariance of the trace, and the definition (2), one recovers (4). Upon verifying and utilizing that $\overline{c_{j}^{*} c_{k} c_{m}^{*} c_{n}}=\left(\delta_{j k} \delta_{m n}+\right.$ $\delta_{j n} \delta_{k m}$ ), a similar calculation yields (5) (analogous calculations can also be found, e.g., in Refs. [7, 8]).

Considering $\operatorname{Tr}\left\{C_{1}^{\dagger} C_{2}\right\}$ as a scalar product between two arbitrary linear (but not necessarily Hermitian) operators $C_{1,2}: \mathcal{H} \rightarrow \mathcal{H}$, the Cauchy-Schwarz inequality takes the form $\left|\operatorname{Tr}\left\{C_{1}^{\dagger} C_{2}\right\}\right|^{2} \leq \operatorname{Tr}\left\{C_{1}^{\dagger} C_{1}\right\} \operatorname{Tr}\left\{C_{2}^{\dagger} C_{2}\right\}$. Choosing $C_{1}=B \rho$ and $C_{2}=\rho B$, it follows that $\operatorname{Tr}\left\{(\rho B)^{2}\right\}=$ 
$\operatorname{Tr}\left\{C_{1}^{\dagger} C_{2}\right\}$ and that both $\operatorname{Tr}\left\{C_{1}^{\dagger} C_{1}\right\}$ and $\operatorname{Tr}\left\{C_{2}^{\dagger} C_{2}\right\}$ can be rewritten as $\operatorname{Tr}\left\{\rho^{2} B^{2}\right\}$. With (5) we thus obtain

$$
\sigma_{B}^{2} \leq \operatorname{Tr}\left\{\rho^{2} B^{2}\right\} .
$$

Evaluating the trace by means of the eigenbasis of $B$, one finds that

$$
\sigma_{B}^{2} \leq\|B\|^{2} \operatorname{Tr}\left\{\rho^{2}\right\},
$$

where $\|B\|$ is the operator norm introduced above (4), and where $\operatorname{Tr}\left\{\rho^{2}\right\}$ is the purity of the density operator $\rho$ from (2).

In the following, we restrict ourselves to operators $R$ in (11), (2) with the property that

$$
\operatorname{Tr}\left\{\rho^{2}\right\} \ll 1
$$

for reasons which will become obvious immediately.

Choosing for $B$ the identity operator, it follows from (3)-(5) that

$$
\overline{\frac{\langle\phi \mid \phi\rangle}{(\langle\phi \mid \phi\rangle-1)^{2}}=1,} \operatorname{Tr}\left\{\rho^{2}\right\} .
$$

With (10) we can conclude that the overwhelming majority of all vectors $|\phi\rangle$ in (11) have norms very close to unity.

Note that the variance $\sigma_{B}^{2}$ in (5) vanishes for $B=0$, and is generically positive for $B=\mathbb{1}$ according to (12). More generally, $\sigma_{B}^{2}$ is not invariant when replacing $B$ in (5) by $B+b \mathbb{1}$ with $b \in \mathbb{R}$. The reason for this quite unusual behavior is that the norm of the vectors in (1D) is not fixed but rather exhibits random fluctuations.

Next, we consider any given $|\phi\rangle$ in (1) as an initial state at time $t=0$, which then evolves according to the timedependent Schrödinger equation into the state $|\phi(t)\rangle=$ $U_{t}|\phi\rangle$ after a time $t$ has elapsed, where $U_{t}$ is the unitary quantum-mechanical time-evolution operator. For a time independent Hamiltonian $H$, the propagator $U_{t}$ takes the simple form $\exp \{-i H t / \hbar\}$, but in full generality, also any explicitly time-dependent Hamiltonian $H(t)$ is admitted (see also Appendix @). In particular, the system is not required to exhibit equilibration or thermalization in the long-time limit.

Going over from the Schrödinger to the Heisenberg picture of quantum mechanics, the corresponding expectation value for an arbitrary observable $O$ (of finite operator norm, see above) at an arbitrary but fixed time $t$ thus follows as

$$
\begin{aligned}
& \langle\phi(t)|O| \phi(t)\rangle=\left\langle\phi\left|O_{t}\right| \phi\right\rangle, \\
& O_{t}:=U_{t}^{\dagger} O U_{t},
\end{aligned}
$$

where we ignore for the time being that the vector $|\phi(t)\rangle$ may not be normalized. Choosing for $B$ the operator $O_{t}$ we can conclude from (4), (5), (9), and (10) that the vast majority of all vectors $|\phi\rangle$ in (10) exhibit expectation values very close to the mean value $\overline{\left\langle\phi\left|O_{t}\right| \phi\right\rangle}$.
Until now, the vectors $|\phi\rangle$ in (1) are in general not normalized. But, as seen below (12), the vast majority among them is almost of unit length. Hence, if we replace every given $|\phi\rangle$ in (1D) by its strictly normalized counterpart

$$
|\psi\rangle:=\langle\phi \mid \phi\rangle^{-1 / 2}|\phi\rangle,
$$

then the "new" expectation values $\langle\psi(t)|O| \psi(t)\rangle$ will mostly remain very close to the "old" ones, i.e., to $\langle\phi(t)|O| \phi(t)\rangle$ (since $\langle\psi(t)|O| \psi(t)\rangle=\langle\phi(t)|O| \phi(t)\rangle /\langle\phi \mid \phi\rangle$ and $\langle\phi \mid \phi\rangle \simeq 1)$. The latter, in turn, are close to $\overline{\left\langle\phi\left|O_{t}\right| \phi\right\rangle}$ for most $|\phi\rangle$ 's (see below (14)). According to (4),,$\overline{\left\langle\phi\left|O_{t}\right| \phi\right\rangle}$ can be rewritten as $\operatorname{Tr}\left\{\rho O_{t}\right\}$ and hence as $\operatorname{Tr}\{\rho(t) O\}$, where

$$
\rho(t):=U_{t} \rho U_{t}^{\dagger}
$$

is the time-evolved density operator. Altogether, we thus can conclude that

$$
\langle\psi(t)|O| \psi(t)\rangle \simeq \operatorname{Tr}\{\rho(t) O\}
$$

is fulfilled in very good approximation for most normalized vectors $|\psi\rangle$ in (15). A fully quantitative version of the latter approximative statement in terms of the small parameter $\operatorname{Tr}\left\{\rho^{2}\right\}$ from (10) can be readily obtained by a similar line of reasoning as in Sec. III.C of Ref. [12].

Note that for any given $t$ there may still be a small set of "untypical" initial states $|\psi\rangle$, for which (17) is a bad approximation. Moreover, this set will usually be different for different time points $t$. Likewise, for any given observable $O$, the small set of untypical states $|\psi\rangle$ will in general be different. Apart from that, the main implication of (17) is that initial states $|\psi\rangle$, randomly sampled according to (11) and (15), are very likely to exhibit very similar expectation values at the initial time $t=0$ and also at any later time, a property which was named dynamical typicality in Ref. [5]. Formally, this typical time-evolution is given by the right hand side of (17), but its explicit, quantitative evaluation is usually very difficult and not further pursued here.

\section{BASIC PROPERTIES OF $R$ AND $\rho$}

So far, the only restriction regarding the choice of $R$ in (11) is that $\rho$ from (2) must satisfy (3) and (10). Two further basic properties of $R$ are:

(i) Since the ensemble of vectors $|\phi\rangle$ in (1) is invariant under arbitrary unitary transformations $U: \mathcal{H} \rightarrow \mathcal{H}$ of the basis $\left|\chi_{n}\right\rangle$ (see below (31)), the same applies to the ensemble of vectors $|\psi\rangle$ in (15). In other words, $R$ and $R^{\prime}:=R U$ lead to identical dynamical typicality properties for arbitrary $U$. (ii) According to textbook linear algebra, any linear operator $R: \mathcal{H} \rightarrow \mathcal{H}$ can be written in the form $R=G U$ with a positive semidefinite Hermitian operator $G$ and a unitary operator $U$ (so-called left polar decomposition). 
Due to (i) and (ii), we can and will restrict ourselves Hermitian operators $R$, i.e.,

$$
R=R^{\dagger}
$$

without any loss of generality. Moreover, we can assume that $R$ is a strictly positive Hermitian operator whenever this is convenient (which is often but not always the case). The reason is as follows: The possibility to choose $R$ positive semidefinite follows from the polar decomposition mentioned in (ii) above. Furthermore, by choosing for $\left|\chi_{n}\right\rangle$ in (11) the eigenvectors of $R$ one sees that vanishing eigenvalues of $R$ are trivial and can be excluded right from the beginning by properly restricting the considered Hilbert space $\mathcal{H}$.

Denoting the eigenvalues and eigenvectors of the density operator $\rho$ by $p_{n}$ and $|n\rangle$, i.e.,

$$
\rho=\sum_{n=1}^{N} p_{n}|n\rangle\langle n|
$$

it follows that $p_{n} \geq 0, \sum_{n=1}^{N} p_{n}=1$ (see also (3i)), and

$$
\operatorname{Tr}\left\{\rho^{2}\right\}=\sum_{n=1}^{N} p_{n}^{2} .
$$

Moreover, the relation between $R$ and $\rho$ may be considered as one-to-one in the following sense: For any given $\rho$, there exist exactly one Hermitian, semipositive $R$, which reproduces $\rho$ via (2), namely

$$
R=\rho^{1 / 2}=\sum_{n=1}^{N} \sqrt{p_{n}}|n\rangle\langle n| .
$$

Moreover, for every other $R$, which yields the same $\rho$ in (21), the resulting random vector ensemble in (1) is indistinguishable from the ensemble generated by (21).

In the last identity in (21), we adopted the usual definition that for an arbitrary function $f(x): \mathbb{R} \rightarrow \mathbb{R}$ (here $f(x)=x^{1 / 2}$ ) and any Hermitian operator $C: \mathcal{H} \rightarrow \mathcal{H}$ with eigenvalues $c_{n}$ and eigenvectors $\left|\varphi_{n}\right\rangle$ (here $p_{n}$ and $|n\rangle)$, the operator $f(C): \mathcal{H} \rightarrow \mathcal{H}$ is defined as

$$
f(C):=\sum_{n=1}^{N} f\left(c_{n}\right)\left|\varphi_{n}\right\rangle\left\langle\varphi_{n}\right| .
$$

Focusing on strictly positive $R$ 's (see below (18)), it follows from (21) that $p_{n}>0$ for all $n$. As a consequence, also $\rho$ in (19) is a positive operator with a well-defined inverse, namely

$$
\rho^{-1}=\sum_{n=1}^{N} p_{n}^{-1}|n\rangle\langle n| .
$$

\section{PRECONDITIONS FOR DYNAMICAL TYPICALITY}

Until now, only the pure states $|\phi\rangle$ from (1) and their normalized counterparts $|\psi\rangle$ from (15) were of immediate physical relevance, while the density operator $\rho$ from (2) was mainly an auxiliary formal tool. Additional insight into the physical meaning of $\rho$ can be gained via the precondition (10) for dynamical typicality:

As observed in [13], the so-called effective dimension $d_{\text {eff }}:=1 / \operatorname{Tr}\left\{\rho^{2}\right\}$ tells us, how many pure states contribute appreciably to the mixture $\rho$. Indeed, one readily finds - similarly as in (4) - that $\overline{|\phi\rangle\langle\phi|}=\rho$, i.e., if this average were dominated by just a few pure states, then the purity $\operatorname{Tr}\left\{\rho^{2}\right\}$ of the mixture $\rho$ could not be very small. More specifically, if $p_{n}=1 / M$ for $M$ of the indices $n$ in (19), and $p_{n}=0$ for all the others, then $d_{\text {eff }}=M$, and the $|\phi\rangle$ in (10) arise by sampling vectors in an unbiased way within an $M$-dimensional subspace of $\mathcal{H}$. In other words, $d_{\text {eff }}$ quantifies the "diversity" of random vectors $|\phi\rangle$ contributing to $\rho$, and (10) ensures that the ensemble of random vectors in (10) is still "reasonably large".

So far, (10) represents a sufficient condition for the dynamical typicality properties established in Sec. III. Next we turn to the question in how far this condition is necessary for dynamical typicality. Our first remark is that for some operators $B$ (e.g. the identity), dynamical typicality is trivially fulfilled, independently of whether $\operatorname{Tr}\left\{\rho^{2}\right\}$ satisfies (10) or not. Hence our goal will be to show that if $\operatorname{Tr}\left\{\rho^{2}\right\}$ is not small then there exist at least one $B$ for which dynamical typicality breaks down.

Taking into account (19) and (20), one readily infers that

$$
\begin{aligned}
& p_{\text {max }}^{2} \leq \operatorname{Tr}\left\{\rho^{2}\right\} \leq p_{\max } \\
& p_{\text {max }}:=\max _{n} p_{n} .
\end{aligned}
$$

In other words $p_{\max }$ is the largest eigenvalue of $\rho$. From (24) we can conclude that $\operatorname{Tr}\left\{\rho^{2}\right\}$ is small if and only if $p_{\max }$ is small, i.e., condition (10) is equivalent to

$$
p_{\max } \ll 1 \text {. }
$$

Next we assume that (10) and hence (26) is not satisfied, i.e. there is at least one $p_{n}$, say $p_{1}$, which is not small (compared to unity). Choosing $\left|\chi_{n}\right\rangle:=|n\rangle$ in (1), $B:=|1\rangle\langle 1|$, and observing (15), (21), it follows that

$$
\begin{aligned}
\langle\psi|B| \psi\rangle & =\frac{p_{1}\left|c_{1}\right|^{2}}{\sum_{n=1}^{N} p_{n}\left|c_{n}\right|^{2}}=\frac{1}{1+z}, \\
z & :=\frac{\sum_{n=2}^{N} p_{n}\left|c_{n}\right|^{2}}{p_{1}\left|c_{1}\right|^{2}}
\end{aligned}
$$

As said below (10), the $c_{n}$ are independent, normally distributed (complex) random numbers. Dynamical typicality means that the expectation values in (27) must be very similar for most realizations of those random numbers. This is trivially the case if $p_{1}=1$ (and hence $p_{n}=0$ for all $n \geq 2$ ). One readily sees that this is not a special feature of our specific choice of $B$ in (27) but rather applies for arbitrary $B$. Similar conclusions remain true as long as $p_{1}$ is very close to unity. In other words, yet 
another sufficient condition (besides (26)) for dynamical typicality is

$$
1-p_{\max } \ll 1 .
$$

Once again, one can infer from (24) that (29) is equivalent to

$$
1-\operatorname{Tr}\left\{\rho^{2}\right\} \ll 1 .
$$

If $p_{1}$ is neither close to zero nor close to unity, then one can infer that $z$ in (28) is a random number, which is typically neither very small nor very large and exhibits non-negligible random fluctuations. Hence, also the expectation value in (27) cannot be almost equal for most realizations of the $c_{n}$ 's, i.e., dynamical typicality is violated.

In conclusion, any of the four conditions (10), (26), (29), and (30) is sufficient for dynamical typicality. In turn, if (10) or (26) and simultaneously (29) or (30) are violated, then dynamical typicality breaks down. However, cases to which (29) or (30) apply are of very limited physical interest and henceforth ignored. In conclusion, either of the two conditions (10) or (26) can thus be considered as necessary and sufficient for dynamical typicality.

\section{PHYSICAL OBSERVABLES AND MEASUREMENT OUTCOMES}

As already pointed out at the beginning of Sec. III. the considered Hilbert space $\mathcal{H}$ may be either of infinite or of large but finite dimensionality. In the first part of this section, we mainly have in mind the latter case. The last part applies to both cases.

Even in case of a finite-dimensional $\mathcal{H}$, the original Hilbert space $\mathcal{H}^{\prime}$ of the actual system of interest is a priori often of infinite dimension and the actual observables $O^{\prime}$ are Hermitian operators on $\mathcal{H}^{\prime}$. The same applies to the Hamiltonian $H^{\prime}(t)$, which in general may be explicitly time-dependent (see above (13)).

In contrast to this original setup, a Hilbert space $\mathcal{H}$ with finite dimensionality $N$ as introduced above (10) usually represents an energy shell, i.e., it is spanned by $N$ eigenvectors of $H^{\prime}(0)$, whose eigenvalues are contained in an energy interval, which is microscopically large (thus $N$ is large) but macroscopically small (well defined system energy). Denoting by $P^{\prime}$ the projector from $\mathcal{H}^{\prime}$ onto $\mathcal{H}$, all normalized pure states $|\psi\rangle$ in (10), (15) thus satisfy $P^{\prime}|\psi\rangle=|\psi\rangle$ and $\left\langle\psi\left|O^{\prime}\right| \psi\right\rangle=\langle\psi|O| \psi\rangle$ with $O:=P^{\prime} O^{\prime} P^{\prime}$. The same properties apply to $H^{\prime}(0)$ (instead of $O^{\prime}$ ). For time independent Hamiltonians, i.e. $H^{\prime}(t)=H^{\prime}(0)$ for all $t$, it is therefore sufficient to focus on the Hilbert space $\mathcal{H}$ and on observables $O: \mathcal{H} \rightarrow \mathcal{H}$. This fact has been tacitly anticipated in Sec. III Some additional formal subtleties in the case of explicitly time-dependent Hamiltonians are worked out in Appendix A.

It should be emphasized that the eigenvalues and eigenvectors of the original (physical) observables $O^{\prime}$ may in general be rather different from those of the auxiliary Hermitian operators $O$. In particular, if $Q^{\prime}: \mathcal{H}^{\prime} \rightarrow \mathcal{H}^{\prime}$ is a projector, then

$$
Q:=P^{\prime} Q^{\prime} P^{\prime}
$$

is in general no longer a projector.

Note that $\left[O^{\prime}, H^{\prime}(0)\right]=0$ (commutator) implies $[O, H(0)]=0$ (conserved quantity), but for the rest, commutators of primed and unprimed operators are in general unrelated.

Adopting the simplest and most common interpretation of quantum mechanics, the outcome of a measurement process will be one of the eigenvalues of the observable $O^{\prime}$ under consideration. Both the set of possible measurement outcomes and their probabilities are usually different for $O^{\prime}$ and $O$. Only the mean values happen to coincide for all $|\psi\rangle \in \mathcal{H}$, while for instance the second moment $\left\langle\psi\left|\left(O^{\prime}\right)^{2}\right| \psi\right\rangle$ is usually (unless $O^{\prime}=O$ ) larger than $\left\langle\psi\left|O^{2}\right| \psi\right\rangle$, and likewise for the variances (this follows from the Cauchy-Schwarz inequality). Nevertheless, higher moments and thus the entire statistics of the measurement outcomes of the physical observable $O^{\prime}$ are still accessible solely by means of suitable auxiliary operators: Namely, the $k$-th moment $\left\langle\psi\left|\left(O^{\prime}\right)^{k}\right| \psi\right\rangle$ can be recovered as $\langle\psi|\tilde{O}| \psi\rangle$ where the auxiliary operator $\tilde{O}: \mathcal{H} \rightarrow \mathcal{H}$ is given by $P^{\prime}\left(O^{\prime}\right)^{k} P^{\prime}$ (which usually differs from $O^{k}$ ).

After these preparatory considerations, we return to the general case of either finite- or infinite-dimensional Hilbert spaces $\mathcal{H}$ (in particular, $\mathcal{H}$ may or may not agree with $\mathcal{H}^{\prime}$, and likewise for $O$ and $H(t)$ ). Our first observation is that the dynamical typicality results below (17) can also be applied simultaneously to several (possibly auxiliary) observables, provided their number is not too large. Hence, analogous typicality properties remain true in good approximation even for the entire measurement outcome statistics of a given observable $O$. Moreover, if $O$ actually represents an auxiliary observable, then the same conclusion remains true even for the underlying physical observable $O^{\prime}$.

\section{EQUILIBRIUM INITIAL CONDITIONS}

Our next goal is to identify particularly interesting Hermitian operators $R$ in (10), (15) which fulfill (3) and either (10) or (26).

The simplest case arises for time independent Hamiltonians $H$ and operators $R$ of the form $R=f(H)$. Hence, $R$ commutes with the time-evolution operator $U_{t}=\exp \{-i H t / \hbar\}$ (see also above (13)) and thus all statistical properties of the random vector ensemble in (1) are time independent. Accordingly, the random vectors from (11), (15) are called equilibrium initial conditions.

As usual in statistical physics, it is not the single realizations $|\psi\rangle$ in (11), (15), but rather it is the statistical ensemble $\rho$ in (2) (see also (16)) which exhibits equilibrium (steady state) properties. If $\rho$ satisfies (10) or (26), it 
follows that the expectation value in (17) is well approximated by the time independent equilibrium value $\operatorname{Tr}\{\rho O\}$ for most $|\psi(t)\rangle$ 's at any given time point $t \geq 0$. Essentially, this amounts to the original, "non-dynamical" or "canonical" typicality results from [2-4, $[6-10]$.

As a first example we consider the case when the Hilbert space $\mathcal{H}$ amounts to an energy shell and, in particular, is of large but finite dimensionality $N$, see also above Eq. (11) and Sec. VI] Choosing $R=\mathbb{1} / \sqrt{N}$ in (21) yields for $\rho$ in (2) the microcanonical ensemble

$$
\rho_{\mathrm{mc}}:=\mathbb{1} / N,
$$

satisfying (3) and implying $\operatorname{Tr}\left\{\rho^{2}\right\}=1 / N$. Since we assumed $N \gg 1$ it follows that (26) is satisfied. Therefore, $\langle\psi(t)|O| \psi(t)\rangle$ will be very close to the thermal equilibrium value $\operatorname{Tr}\left\{\rho_{\mathrm{mc}} O\right\}$ for most $|\psi(t)\rangle$ 's, where $O$ is an arbitrary but fixed observable and $t$ an arbitrary but fixed time point (also $t=0$ is admitted). As noted in [14, 15], the essential point is that all possible orientations of the orthonormal basis $|n\rangle$ in (1) relative to the eigenbasis of $O$ are sampled with equal probabilities (formally by means of Haar distributed unitary basis transformations). Therefore, instead of considering $|\psi(t)\rangle$ as random and $O$ as arbitrary but fixed, one could equally well consider $|\psi(t)\rangle$ as arbitrary but fixed and the eigenbasis of $O$ as random (the eigenvalues of $O$ and the time point $t$ are also kept fixed). The main conclusion is that for any given $|\psi(t)\rangle$, the vast majority of all observables $O$ yield expectation values $\langle\psi(t)|O| \psi(t)\rangle$ very close to the equilibrium value $\operatorname{Tr}\left\{\rho_{\mathrm{mc}} O\right\}$.

As a second example, we consider the case that $\mathcal{H}$ is the full Hilbert space of the system of interest and, in particular, may be infinite-dimensional, see also above Eq. (11) and Sec. VI] Choosing $R \propto \exp \{-\beta H / 2\}$ in (21) yields via (2) the canonical ensemble

$$
\begin{aligned}
\rho_{\text {can }} & :=Z^{-1} e^{-\beta H}, \\
Z & :=\operatorname{Tr}\left\{e^{-\beta H}\right\},
\end{aligned}
$$

where the exponential functions of $H$ are understood in the sense of (22). Furthermore, condition (26) will be satisfied if $H$ exhibits many eigenvalues $E_{n}$, whose distance to the ground state energy is smaller than the thermal energy $k_{B} T:=1 / \beta$, since many of the eigenvalues $p_{n}=Z^{-1} \exp \left\{-\beta E_{n}\right\}$ of $\rho$ will then be comparable to $p_{\max }$ and hence $p_{\max }$ must be small. Given that generic many-body Hamiltonians exhibit an extremely dense spectrum even at rather low energies, condition (26) will thus be fulfilled for virtually any experimentally realistic temperature $T$.

In conclusion, very accurate approximations for any expectation value in the canonical ensemble can be obtained with extremely high probability by randomly sampling a single pure state $|\psi\rangle$ according to (1), (15) and $R$ as specified above. Moreover, we recall that in (1) any basis $\left|\chi_{n}\right\rangle$ of the Hilbert space under consideration will do.
For practical purposes, it is often convenient to break up the above scheme into two steps. In a first step, instead of working with $R=\gamma \exp \{-\beta H / 2\}$, where $\gamma$ is fixed via (2) and (3), one rather chooses $\gamma=1$. The resulting operator $R=\exp \{-\beta H / 2\}$ can be numerically evaluated very efficiently for instance by means of imaginary time propagator techniques [16]. Obviously, high temperatures (small $\beta$ ) are expected to be particularly easily tractable, however, apart from the above excluded, exceedingly low temperatures, all temperature regimes should be feasible. Moreover, the values of $\langle\phi \mid \phi\rangle$ for the resulting random vectors $|\phi\rangle$ in (11) will no longer be with very high probability close to unity (see below (12)), but rather close the value $Z$ from (34). Since $Z$ is the canonical partition function, one thus also obtains the free energy and all the hence deriving further thermodynamic quantities in the usual way. In a second step, the canonical expectation value of an arbitrary observable $O$ can be obtained from the same random vector $|\phi\rangle$ by evaluating $\langle\phi|O| \phi\rangle /\langle\phi \mid \phi\rangle$. In conclusion, a single typical pure state $|\phi\rangle$ is able to imitate all physically relevant properties of a full fledged canonical equilibrium ensemble!

All these quite remarkable observations have been analytically elaborated and numerically exemplified in a particularly clear and comprehensive work by Sugiura and Shimizu in Ref. [17]. However, many of the key ideas have been theoretically recognized and numerically employed in a considerable number of much earlier works, most notably in Ref. [18], but also in Refs. 19]. Analogous concepts of how to imitate microcanonical and grand canonical ensembles by randomly sampling a single pure state have been worked out in Refs. [9, 20].

If the Hamiltonian is explicitly time-dependent, already such simple choices as $R=f(H(0))$ may lead to quite interesting consequences and applications! The main caveat is the observation below (32) that most observables may yield expectation values close to the equilibrium value for any given $|\psi(t)\rangle[14$, 15]. Therefore, it may be necessary to carefully select a suitable observable in order to "see" any non-trivial effect of the external perturbation, which generates the time-dependence of the Hamiltonian. This may be worthwhile to be explored numerically for specific model systems, e.g. exhibiting a quench dynamics or a periodic time-dependence.

\section{NON-EQUILIBRIUM INITIAL CONDITIONS}

Especially for time independent Hamiltonians, initial states with certain non-equilibrium expectation values are of particular interest. Referring to Sec. IA for a more detailed justification, we henceforth focus on situations where the expectation values of one or several observables $A_{k}(k=1, \ldots, K)$ are known to (approximately) assume certain values $\alpha_{k}$, which differ from the equilibrium expectation values discussed in the preceding subsection. 
A natural way to incorporate those preset expectation values $\alpha_{k}$ is by means of additional constraints on $\rho$ (and thus via (2) or (21) on $R$ ) of the form

$$
\operatorname{Tr}\left\{\rho A_{k}\right\}=\alpha_{k} \text { for } k=1, \ldots, K .
$$

Indeed, taking for granted (10) and setting $t=0$ in (17) implies that $\left\langle\psi\left|A_{k}\right| \psi\right\rangle$ will be close to $\alpha_{k}$ for most initial states $|\psi\rangle$.

Accordingly, our next goal is to find $\rho$ 's which satisfy (35) on top of (3) and (10). First of all, it seems reasonable to assume that the non-equilibrium character of a macroscopic initial state in a real experiment (see also Sec. IIA can be captured by a relatively small number $K$ of non-trivial (non-equilibrium) expectation values $\alpha_{k}$ appearing in (35). Yet, it is quite obvious that there still may exist certain combinations of $A_{k}$ 's and $\alpha_{k}$ 's for which (3) and (35) cannot be satisfied by any (pure or mixed) quantum state $\rho$ :

To begin with, only $\alpha_{k}$ values in between the smallest and the largest eigenvalues of $A_{k}$ are admissible, otherwise (35) cannot be satisfied by any $\rho$.

Furthermore, the operators $\mathbb{1}$ and all the $A_{k}$ 's in (35) must be linearly independent in the following sense: The only real numbers $x_{k}$ which solve the equation

$$
x_{0} \mathbb{1}+\sum_{k=1}^{K} x_{k} A_{k}=0
$$

are $x_{k}=0$. The reason is as follows: If non-trivial solutions of (36) would exist, then at least one $x_{k}$ with $k>0$ must be non-zero, say $x_{1} \neq 0$. With $b_{k}:=-x_{k} / x_{1}$ it follows that $A_{1}=b_{0} \mathbb{1}+\sum_{k=2}^{K} b_{k} A_{k}$ and with (3), (35) that $\alpha_{1}=b_{0}+\sum_{k=2}^{K} b_{k} \alpha_{k}$. If $\alpha_{1}$ fulfills this equality then the constraint (35) for $k=1$ is redundant and can be omitted without any loss of generality. If $\alpha_{1}$ violates this equality, then the problem was ill posed in the first place.

Finally, it should be emphasized that the $A_{k}$ 's in (35) may or may not commute, and that particularly complicated restrictions regarding physically reasonable combinations of the $\alpha_{k}$ 's are expected to arise for noncommuting $A_{k}$ 's. The reasons for such incompatible constraints may or may not be obvious and their systematic exploration goes beyond our present scope. Moreover, while the so far discussion mainly emphasized the mathematical side of the problem, physically speaking such incompatible conditions would indicate either a meaningless (experimentally unrealistic) situation or that the theoretical model was inappropriate in the first place. For all these reasons, we henceforth take for granted that (3) and (35) are satisfiable by at least one density operator $\rho$.

In many cases, already a few constraints of the form (35) may adequately capture the non-equilibrium initial condition at hand. In other cases, the number $K$ of relevant constraints (35) may be quite considerable, for instance in order to describe initial states with complex spatial inhomogeneities. Nevertheless, a number $K$ much smaller than $N$ will usually be sufficient. In particular, the number of parameters (independent matrix elements) specifying $\rho$ is thus much larger than the number of equations (3) and (35). Moreover, (3) and (35) give rise to linear equations for the matrix elements of $\rho$. It follows that - given at least one solution exists generically there actually will be many different solutions $\rho$ of those equations. Likewise, if one of those $\rho$ 's in addition satisfies condition (10), then the same is expected to apply also for many other $\rho$ 's (for instance those within a "close neighborhood"). Moreover, if we denote by $\rho_{1}$ and $\rho_{2}$ two different such solutions, then generically there will exist certain observables $B$ for which the expectation values $\operatorname{Tr}\left\{\rho_{1} B\right\}$ and $\operatorname{Tr}\left\{\rho_{2} B\right\}$ differ by a non-negligible amount. Recalling that the Hamiltonian and hence the unitary time propagator $U_{t}$ in (14) may, in principle, still be chosen largely arbitrary (see also Appendix $\mathrm{A}$ ), the above $B$ may be reproduced by $O_{t}$ via (14) in terms of a quite "harmless" observable $O$ (for instance by one of the $A_{k}$ 's), yielding $\operatorname{Tr}\{\rho B\}$ for the expectation value at time $t$ on the right hand side of (17). An extreme example arises when both $\rho_{1}$ and $\rho_{2}$ satisfy (3), (10), (35), and, in addition, both "secretly" satisfy one more condition of the type (35), say $\operatorname{Tr}\{\rho B\}=\beta$, but with two different values for $\beta$. In this case, dynamical typicality behavior is recovered in both cases, but the typical expectation values are different in the two cases, for instance for the observable $B$ at time $t=0$.

We also note that whenever two different solutions $\rho_{1}$ and $\rho_{2}$ of (35) are at hand, then most of the random vectors associated with $\rho_{1}$ via (1), (15), and (21) must be considered as "untypical" samples of the random vector ensemble associated with $\rho_{2}$.

\section{A. Unbiasedness assumption}

The overall so far conclusion is: Given that (3), (10), and (35) can be satisfied at all, then generically many different $\rho$ 's will do so. While each of them will exhibit dynamical typicality, the predicted typical behavior may quite notably differ between some of them. Hence, the question arises, which $\rho$ is the "right" one, e.g., in order to describe an ensemble of experiments which are repeated (as well as possible) at different times or in different labs (see Sec. ПIA).

In order to uniquely determine the "right" $\rho$ along these lines, additional requirements are clearly indispensable. Here we choose them as announced in Sec. IA (see also above Eq. (11), at the beginning of Sec. VI, and around (32)). Namely, we assume that the initial system energy is known (and reproducible) up to an uncertainty which is small on macroscopic but large on microscopic scales, i.e., the relevant Hilbert space $\mathcal{H}$ is an energy shell, spanned by a large but finite number $N$ of eigenvectors of the (time independent) Hamiltonian $H$. Within this Hilbert space, our random ensemble of vectors $|\psi\rangle$ is defined as usual via (11) and (15). Now our key requirement, 
by which $\rho$ will then be uniquely determined, consist in the following unbiasedness assumption: If two random vectors $|\psi\rangle$ exhibit equal expectation values $\left\langle\psi\left|A_{k}\right| \psi\right\rangle$ for all $k=1, \ldots, K$, then they are realized with equal probability. It is our contention that the situation outlined in Sec. II A is appropriately captured in this way: We know the (approximate) energy of the initial system state $|\psi\rangle$, as well as the (approximate) expectation values of the observables $A_{k}$. Apart from that, no further information about $|\psi\rangle$ is available. It is therefore natural to model the initial states in accordance with the above unbiasedness assumption, e.g., in order to describe many repetitions of the "same" experiment.

Yet another important justification of the above unbiasedness assumption is that the resulting random vectors $|\psi\rangle$ will closely approximate the random vectors with strictly fixed initial conditions from Sec. IXA.

\section{B. Determination of $\rho$}

Our starting point is the following general result, whose detailed derivation is provided in Appendix B: Given an arbitrary but fixed $\rho$, we choose its eigenvectors $|n\rangle$ (cf. (19)) as basis vectors $\left|\chi_{n}\right\rangle$ in (11). With (21) we thus can rewrite (15) as

$$
\begin{aligned}
|\psi\rangle & =\sum_{n=1}^{N} y_{n}|n\rangle, \\
y_{n} & =\frac{\sqrt{p_{n}} c_{n}}{\sqrt{\sum_{n=1}^{N} p_{n}\left|c_{n}\right|^{2}}} .
\end{aligned}
$$

By definition, the probability distribution $\tilde{w}(|\psi\rangle)$ of the vectors $|\psi\rangle \in \mathcal{H}$ is equal to the probability distribution $w(\mathbf{y})$ of the corresponding random numbers $\mathbf{y}:=$ $\left(y_{1}, \ldots, y_{N}\right)$ in (37), whose statistical properties are determined via (38) by those of the $c_{n}$ 's in (11). Referring to Appendix B for the details, the final result is

$$
\begin{aligned}
\tilde{w}(|\psi\rangle) & =w(\mathbf{y})=\mathcal{N} \frac{\delta(\|\psi\|-1)}{\left\langle\psi\left|\rho^{-1}\right| \psi\right\rangle^{N}}, \\
\mathcal{N} & :=\frac{(N-1) !}{2 \pi^{N} \operatorname{det}(\rho)},
\end{aligned}
$$

where $\|\psi\|:=\langle\psi \mid \psi\rangle^{1 / 2}$ is the norm of $|\psi\rangle$ and $\operatorname{det}(\rho):=$ $\prod_{n=1}^{N} p_{n}$ the determinant for $\rho$ from (19). Moreover, we have taken for granted that the considered Hilbert space $\mathcal{H}$ has already been properly adapted as detailed below (18), so that $\rho$ is strictly positive and its inverse in (23) is well defined.

We remark that the same result as in (39) has been obtained (by methods different from those in Appendix B) previously in Ref. 21] (see Appendix therein). Moreover, a closely related result is also contained in Ref. 24] (see equation (18b) therein).

Focusing on $\rho$ 's which satisfy the unbiasedness assumption from Sec. VIIIA the probability in (39) must be equal for all normalized vectors $|\psi\rangle$ with identical values of $a_{\psi}^{k}:=\left\langle\psi\left|A_{k}\right| \psi\right\rangle$. It follows that the quantity $\left\langle\psi\left|\rho^{-1}\right| \psi\right\rangle$ on the right hand side of (39) must be completely determined by the $K$ real numbers $a_{\psi}^{k}$, i.e., there exists a function $h: \mathbb{R}^{K} \rightarrow \mathbb{R}$ with the property that

$$
\left\langle\psi\left|\rho^{-1}\right| \psi\right\rangle=h\left(a_{\psi}^{1}, \ldots, a_{\psi}^{K}\right)
$$

for all normalized vectors $|\psi\rangle$. Under the tacit assumption that the function $h\left(x_{1}, \ldots, x_{K}\right)$ is differentiable at $x_{k}=0(k=1, \ldots, K)$, we can conclude that there must exist real constants $\lambda_{k}:=\partial h(0, \ldots, 0) / \partial x_{k}$ so that

$$
\begin{aligned}
\left\langle\psi\left|\rho^{-1}\right| \psi\right\rangle & =\lambda_{0}+\sum_{k=1}^{K} \lambda_{k}\left\langle\psi\left|A_{k}\right| \psi\right\rangle+\ldots \\
& =\left\langle\psi\left|\left(\lambda_{0} \mathbb{1}+\sum_{k=1}^{K} \lambda_{k} A_{k}\right)\right| \psi\right\rangle+\ldots
\end{aligned}
$$

for all normalized vectors $|\psi\rangle$, where the dots indicate the usual "remainder terms" of higher than linear order in $a_{\psi}^{k}:=\left\langle\psi\left|A_{k}\right| \psi\right\rangle$. It is reasonable to expect that, in general, these higher order contributions cannot be written in the form $\langle\psi|B| \psi\rangle$ for some Hermitian operator $B$, unless they happen to be identically zero. But since all other operators appearing in (42) are Hermitian, those higher order terms must be zero. The remaining equation (42) can only be valid for all $|\psi\rangle$ if the operators on both sides are identical, implying

$$
\rho=\left[\lambda_{0} \mathbb{1}+\sum_{k=1}^{K} \lambda_{k} A_{k}\right]^{-1},
$$

where the right hand side is understood in the sense of (22). An alternative, more rigorous but also more involved derivation of (43) is provided in Appendix C.

The remaining $K+1$ parameters $\lambda_{0}, \ldots, \lambda_{K}$ in (43) must be chosen so that the $K+1$ equations (3) and (35) are fulfilled. Moreover, the resulting solution must satisfy

$$
\lambda_{0} \mathbb{1}+\sum_{k=1}^{K} \lambda_{k} A_{k}>0 .
$$

since $\rho$ was assumed to be a strictly positive operator (see below (401). Regarding the issue whether any solution of those equations exists, similar considerations as below (35) apply. In particular, we again restrict ourselves to situations, for which at least one solution exists. But since the number of parameters is now equal to the number of equations, it is reasonable to expect that this solution will moreover be unique. In the special case $K=1$ this has been rigorously verified in [12]. Finally, one of the $K+1$ equations can always be solved right away so that effectively only $K$ equations with $K$ unknowns remain, see Appendix D

The above findings represent the first main result of our paper. In particular, any $\rho$ which satisfies (3) and 
(35) but is not of the form (43) is biased: Random vectors $|\psi\rangle$ with identical expectation values for all $A_{k}$ are not realized with equal probabilities. If in addition (10) is fulfilled, such a $\rho$ still exhibits dynamical typicality properties, but they may be different from those which an unbiased ensemble would exhibit.

\section{Canonical density operators are biased}

As stated at the beginning of Sec. VIIIA from the physical viewpoint we are mainly interested in the "microcanonical" situation that the relevant Hilbert space $\mathcal{H}$ amounts to an energy shell. However, from the formal viewpoint, our above derived main results remain valid under considerably more general circumstances. In particular, we may consider the special case of a timeindependent system (Hamiltonian $H$ ) with only one single constraint $(K=1)$ of the form $\operatorname{Tr}\{\rho H\}=E$, i.e., $A_{1}:=H$ and $\alpha_{1}:=E$. In this case, one might naively expect that the resulting density operator $\rho$ should be of the "canonical" form $Z^{-1} e^{-\beta H}$ for some suitable $\beta$ and $Z$ (provided $E$ was chosen "reasonable"). On the other hand, such a canonical form is clearly incompatible with (43). The reason is that such a canonical density operator $\rho$ violates the unbiasedness assumption from Sec. VIII A. At first glance, this may appear hard to believe, but upon closer inspection one realizes that one actually cannot come up with a good argument why a canonical $\rho$ should generate an unbiased ensemble of random vectors. In fact, one can readily construct simple examples which explicitly show that such ensembles are biased. For more details on this issue see also [22].

\section{ALTERNATIVE ENSEMBLES}

We start by recalling the three main properties of the normalized random vectors $|\psi\rangle$ in (15) or (37) when the density operator is of the specific form (43) and satisfies (3), (10), and (35): (i) Dynamical typicality: For most of the time-evolved states $|\psi(t)\rangle$, the expectation values $\langle\phi(t)|O| \phi(t)\rangle$ are close to the ensemble average in (17) for any given observable $O$ and time point $t$. (ii) Initial disequilibrium: The expectation values $\left\langle\psi\left|A_{k}\right| \psi\right\rangle$ at time $t=0$ are almost equal to the preset ensemble averages in (35) for most of the $|\psi\rangle$ 's. (iii) Unbiasedness: All vectors $|\psi\rangle$ with exactly identical expectation values $\left\langle\psi\left|A_{k}\right| \psi\right\rangle$ for all $k=1, \ldots, K$ are realized with equal probability.

Besides this random vector ensemble, henceforth denoted as $E$, the following subsections explore two alternative ensembles of normalized random vectors, which will be named $S$ and GAP, and which exhibit the same general properties (i)-(iii) as E. More precisely, with respect to (i) and (ii), all their statistical properties are practically indistinguishable from those of $E$, though in principle there remain certain, extremely small deviations, while (iii) is strictly obeyed by all three ensembles.

\section{A. Ensembles with strictly fixed initial conditions}

We consider the ensemble $S$ of all normalized vectors $|\psi\rangle$, whose expectation values $\left\langle\psi\left|A_{k}\right| \psi\right\rangle$ are strictly (hence the "S") equal to some preset values $x_{k}$, i.e.,

$$
\left\langle\psi\left|A_{k}\right| \psi\right\rangle=x_{k} \text { for } k=1, \ldots, K \text {. }
$$

For the rest, all those normalized $|\psi\rangle \in S$ are again realized with equal probability. Note that a priori, the $x_{k}$ in (45) are independent of the $\alpha_{k}$ in (35), but it will obviously be of foremost interest to compare ensembles $E$ and $S$ with $x_{k} \simeq \alpha_{k}$. For $K=1$, the ensemble $S$ and many of its principal properties have been previously explored by Fine [22] and by Müller et al. [23], see also 12].

Put differently, $S$ fulfills the same property (iii) as before in conjunction with a property, which is stronger than (ii). An interesting question is therefore, whether $S$ still exhibits dynamical typicality (property (i)). In the following, we will argue that this is indeed the case. Moreover, when $x_{k} \simeq \alpha_{k}$ for all $k$ then the corresponding typical expectation values $\langle\psi(t)|O| \psi(t)\rangle$ for the two ensembles $S$ and $E$ will even be very similar to each other. In other words, most random vectors from one ensemble closely imitate the behavior of most random vectors from the other ensemble.

Conceptually, the ensemble $S$ seems in many respects physically more sensible than $E$. For instance, its properties are in some way "cleaner" (more precisely specified) and it contains "fewer" elements $|\psi\rangle$, so that statements about "most" $|\psi\rangle$ are in a certain sense "stronger". On the other hand, finding actual samples $|\psi\rangle$ of $S$ in practice (e.g. by numerical means), seems considerably more demanding than sampling random vectors $|\psi\rangle$ from $E$. Therefore, it is very useful to know that a typical $|\psi\rangle \in E$ imitates very well the properties of a typical $|\psi\rangle \in S$.

Similarly as in (13) and (14), taking into account the time-evolution of $|\psi\rangle$ can be avoided by exploring the typicality properties of the expectation values $\langle\psi|B| \psi\rangle$ for an arbitrary but fixed observable $B$. Furthermore, the joint probability density $P_{E}(b, \mathbf{x})$ that a random vector $|\psi\rangle \in E$ simultaneously satisfies $\langle\psi|B| \psi\rangle=b$ and $\left\langle\psi\left|A_{k}\right| \psi\right\rangle=x_{k}$ for arbitrary but fixed values of $b$ and $\mathbf{x}:=\left(x_{1}, \ldots, x_{K}\right)$ can be written by means of the probability distribution from (39) as

$$
\begin{aligned}
P_{E}(b, \mathbf{x}) & =\int d \mathbf{y} w(\mathbf{y}) \delta\left(b_{\psi}-b\right) \prod_{k=1}^{K} \delta\left(a_{\psi}^{k}-x_{k}\right) \\
b_{\psi} & :=\langle\psi|B| \psi\rangle \\
a_{\psi}^{k} & :=\left\langle\psi\left|A_{k}\right| \psi\right\rangle .
\end{aligned}
$$

Exploiting in (39) the special form (43) of $\rho$ for the ensemble $E$ under consideration, we can rewrite (46) as

$$
\begin{aligned}
P_{E}(b, \mathbf{x}) & =g(\mathbf{x}) \int d \mathbf{y} \delta(\|\psi\|-1) \delta\left(b_{\psi}-b\right) \prod_{k=1}^{K} \delta\left(a_{\psi}^{k}-x_{k}\right)(49) \\
g(\mathbf{x}) & :=\mathcal{N}\left(\lambda_{0}+\sum_{k=1}^{K} \lambda_{k} x_{k}\right)^{-N},
\end{aligned}
$$


where the normalization constant $\mathcal{N}$ is defined in (40). As usual, the (marginal) probability distribution for the expectation values of $B$ alone follows by integrating out the variables $\mathbf{x}$, i.e.,

$$
P_{E}(b):=\int d \mathbf{x} P_{E}(b, \mathbf{x})
$$

Similarly, the (joint) probability distribution for the expectation values of the $A_{k}$ 's alone is

$$
\tilde{P}_{E}(\mathbf{x}):=\int d b P_{E}(b, \mathbf{x}) .
$$

The only purpose of the tilde symbol is to better distinguish the two quantities on the left hand side of (51) and of (52). Finally, the conditional probability $P_{E}(b \mid \mathbf{x})$, i.e. the probability that $\langle\psi|B| \psi\rangle$ assumes the value $b$, given that $\left\langle\psi\left|A_{k}\right| \psi\right\rangle=x_{k}$ for all $k$, is defined as

$$
P_{E}(b \mid \mathbf{x}):=\frac{P_{E}(b, \mathbf{x})}{\tilde{P}_{E}(\mathbf{x})} .
$$

Introducing (49) into (52) and (53) yields

$$
P_{E}(b \mid \mathbf{x})=\frac{\int d \mathbf{y} \delta(\|\psi\|-1) \delta\left(b_{\psi}-b\right) \prod_{k=1}^{K} \delta\left(a_{\psi}^{k}-x_{k}\right)}{\int d \mathbf{y} \delta(\|\psi\|-1) \prod_{k=1}^{K} \delta\left(a_{\psi}^{k}-x_{k}\right)}
$$

One readily sees that this is exactly the probability distribution for the expectation values of $B$ when the $|\psi\rangle$ 's are randomly sampled from the ensemble $S$ with constraints (45), symbolically indicated as

$$
P_{S}(b \mid \mathbf{x})=P_{E}(b \mid \mathbf{x}) .
$$

Throughout this section, the $\alpha_{k}$ 's in (35) are tacitly considered as arbitrary but fixed. However, if we would consider them as variable, then $P_{E}(b, \mathbf{x})$ would depend on the $\alpha_{k}$ 's via $g(\mathbf{x})$ in (49). As a consequence, also $P_{E}(b)$ in (51) and $\tilde{P}_{E}(\mathbf{x})$ in (52) are generically expected to depend on the $\alpha_{k}$ 's. On the other hand, $P_{E}(b \mid \mathbf{x})$ in (53) quite remarkably is independent of the $\alpha_{k}$ 's, as can be inferred from (54). Due to (55), the same property applies to $P_{S}(b \mid \mathbf{x})$.

Combining (51), (53), and (55) yields

$$
P_{E}(b):=\int d \mathbf{x} \tilde{P}_{E}(\mathbf{x}) P_{S}(b \mid \mathbf{x}) .
$$

Taking for granted that the density operator $\rho$, corresponding to the ensemble $E$ at hand, satisfies (10), we know that dynamical typicality applies. In particular, the distribution of the expectation values of $B$ on the left hand side in (56) must be sharply peaked about the mean value $\operatorname{Tr}\{\rho B\}$. (Closer inspection reveals that the distribution is actually very close to a Gaussian if $K=1$ [21], and it is reasonable to expect that the same remains true for $K>1$.) Likewise, the joint distribution $\tilde{P}_{E}(\mathbf{x})$ of the expectation values of the $A_{k}$ 's must be very narrowly peaked about the corresponding mean values
$\operatorname{Tr}\left\{\rho A_{k}\right\}$, which in turn are equal to the $\alpha_{k}$ 's according to (35). Is it possible that the convolution of this narrow distribution $\tilde{P}_{E}(\mathbf{x})$ with $P_{S}(b \mid \mathbf{x})$ on the right hand side of (56) can lead to a very narrow distribution $P_{E}(b)$ on the left hand side, without $P_{S}(b \mid \mathbf{x})$ being a very sharply peaked function of $b$ for any given vector $\mathbf{x}$ ? In principle, this is clearly possible as long as the $\mathbf{x}$ values with non-narrow functions $P_{S}(b \mid \mathbf{x})$ only contribute with small weights $\tilde{P}_{E}(\mathbf{x})$ in (56). In practice, it seems reasonable to expect that the main features of the function (54) of $b$ (e.g., its mean and variance) should not abruptly change upon small variations of the parameters x. (Again, for $K=1$ this can even be rigorously verified [12]). Taking this assumption for granted, one can approximate $P_{S}(b \mid \mathbf{x})$ by $P_{S}(b \mid \boldsymbol{\alpha})$ for all $\mathbf{x}$ with non-negligible weights $\tilde{P}_{E}(\mathbf{x})$ in (56) , where $\boldsymbol{\alpha}:=\left(\alpha_{1}, \ldots, \alpha_{K}\right)$, i.e.,

$$
P_{S}(b \mid \mathbf{x}) \simeq P_{S}(b \mid \boldsymbol{\alpha}) \text { if } \mathbf{x} \simeq \boldsymbol{\alpha} .
$$

We thus can infer from (56) the approximation

$$
P_{E}(b) \simeq P_{S}(b \mid \boldsymbol{\alpha}) .
$$

These approximations (57) and (58) are tantamount to the announced result that the two random vector ensembles $E$ and $S$ with $x_{k} \simeq \alpha_{k}$ exhibit very similar typicality properties.

\section{B. The Gaussian adjusted projected ensemble}

Our starting point is an arbitrary but fixed density operator $\rho$ in (19). The corresponding, so-called Gaussian adjusted (GA) ensemble [24] consist of random vectors

$$
|\varphi\rangle=\sum_{n=1}^{N} v_{n}|n\rangle
$$

where the random variables $\mathbf{v}:=\left(v_{1}, \ldots, v_{N}\right) \in \mathbb{C}^{N}$ may equivalently be considered as $2 N$-dimensional real vectors (see also Appendix $\mathrm{B}$ ), and whose probability distribution is given by

$$
w_{G A}(\mathbf{v}):=\frac{1}{\pi^{N} \operatorname{det}(\rho)}\|\mathbf{v}\|^{2} \exp \left\{-\sum_{n=1}^{N} \frac{\left|v_{n}\right|^{2}}{p_{n}}\right\} .
$$

The word "Gaussian" refers to the exponential term in (60), while the word "adjusted" refers to the factor $\|\mathbf{v}\|^{2}$, whose purpose will become clear later. Similarly as in (11), the norm of the vector in (59) is itself a random variable.

The so-called Gaussian adjusted projected (GAP) ensemble amounts - similarly as in (15) - to the normalized counterparts of (59), hence the name "projected" [24, 25]. In other words the GAP ensemble consists of normalized vectors $|\psi\rangle$ of the form (37), where, instead of (39), the probability distribution of the random variables $\mathbf{y}:=\left(y_{1}, \ldots, y_{N}\right)$ now derives from that of the v's in (60) according to (see also Appendix [B)

$$
w_{G}(\mathbf{y}):=\int d \mathbf{v} w_{G A}(\mathbf{v}) \delta(\mathbf{y}-\mathbf{v} /\|\mathbf{v}\|) .
$$


Similarly as in (39), the probability distribution $\tilde{w}_{G}(|\psi\rangle)$ of the vectors $|\psi\rangle$ is identified with $w_{G}(\mathbf{y})$, and similarly as in the Appendix B, the integral in (61) can be evaluated to yield

$$
\begin{aligned}
\tilde{w}_{G}(|\psi\rangle) & =w_{G}(\mathbf{y})=\mathcal{N}_{G} \frac{\delta(\|\psi\|-1)}{\left\langle\psi\left|\rho^{-1}\right| \psi\right\rangle^{N+1}}, \\
\mathcal{N}_{G} & :=\frac{N !}{2 \pi^{N} \operatorname{det}(\rho)} .
\end{aligned}
$$

Denoting the average over the above specified GAP ensemble by an overbar, it has been shown in Refs. 24, 25] that

$$
\begin{aligned}
\bar{B} & :=\overline{\langle\psi|B| \psi\rangle}=\operatorname{Tr}\{\rho B\} \\
\sigma_{B}^{2} & :=\overline{(\langle\psi|B| \psi\rangle-\overline{\langle\psi|B| \psi\rangle})^{2}} \\
& \leq \Delta_{B}^{2} \operatorname{Tr}\left\{\rho^{2}\right\}\left(1+\mathcal{O}\left(\sqrt{\operatorname{Tr}\left\{\rho^{2}\right\}}\right)\right),
\end{aligned}
$$

where $B: \mathcal{H} \rightarrow \mathcal{H}$ may be any Hermitian operator, and where $\Delta_{B}$ denotes the range of $B$, i.e., the difference between its largest and smallest eigenvalues.

These results are remarkably similar to those in (4) and (5). The main difference is that the norm of the vectors $|\phi\rangle$ in (4) and (5) is a random variable, while the vectors $|\psi\rangle$ in (64) and (65) are strictly normalized. This is the main purpose of the "adjustment" factor $\|\mathbf{v}\|^{2}$, as announced below (60). Note also that, as discussed around (17), the normalized counterparts of the $|\phi\rangle$ 's from (15) satisfy a relations of the form (64) only approximately, and under the additional proviso that $\rho$ satisfies the extra condition (10). In contrast, (64) is an exact identity for arbitrary density operators $\rho$ and arbitrary $N$. Likewise, the term $\mathcal{O}\left(\sqrt{\operatorname{Tr}\left\{\rho^{2}\right\}}\right)$ on the right hand side of (65) can be replaced by a rigorous but somewhat lengthy upper bound, see equation (75) in [25].

Under our usual premises that $\rho$ satisfies (3), (10), and (35), the random vector ensemble $E$ from (11), (15) [or from (37), (38)] is thus practically indistinguishable from the GAP ensemble. In particular, if $\rho$ is of the form (43), then both ensembles exhibit the properties (i)-(iii) from the beginning of this Section. Moreover the close similarity is directly indicated by the explicit expressions for the probability distributions corresponding to $E$, as given in (39), and corresponding to GAP as given in (62). Obviously the only relevant difference is the exponent of the denominator, which is $N$ for $E$ and $N+1$ for GAP. For sufficiently large $N$ this difference is relatively minor.

\section{Discussion}

In comparison with the ensemble $E$ (defined at the beginning of this section), a key difference of the $S$ and GAP ensembles is that when writing the corresponding random vectors in the form (37) then - unlike in (38) - the coefficients $y_{n}$ can no longer be conveniently realized by straightforward sampling of independent random variables. In particular, there exists no $R$ which could generate those ensembles via (11) and (15). Nevertheless, both of them still amount to perfectly well-defined ensembles of normalized random vectors.

In other words, the first main advantage of the ensemble $E$ is that it can be easily implemented numerically. A second advantage is that it is also relatively convenient (compared with the other two ensembles) for analytical investigations, as demonstrated with our present paper.

The main virtue of the $S$ ensemble is the conceptual appeal of its "clean" and "natural" definition (see below (45) ).

The main advantage of the GAP ensemble is that it often admits exact analytical statements (in contrast to bounds or large $N$ asymptotics).

For the rest, all three ensemble result in practically indistinguishable expectation value statistics (dynamical typicality properties etc.) for sufficiently large dimensions $N$.

Overall, the main conclusion of this section may be summarized as follows: The conceptually most "natural" ensemble $S$, realizing with equal probability all pure states $|\psi\rangle$ with $K$ (strictly) preset expectation values $\left\langle\psi\left|A_{k}\right| \psi\right\rangle$, is hard to access numerically as well as analytically. On the other hand, the essential properties of that ensemble $S$ are practically perfectly imitated by the much more convenient ensemble $E$, which is readily accessible via (11), (15), (21), (43). Most importantly, demonstrating dynamical typicality properties directly for the $S$ ensemble is expected to be much more difficult than along our present, "indirect" approach via the $E$ ensemble.

\section{TYPICALITY OF EQUILIBRATION}

In this section, we focus on isolated systems, described by a time independent Hamiltonian $H$ with eigenvalues $E_{n}$ and eigenvectors $\left|\chi_{n}\right\rangle$ (see also beginning of Sec. III and Sec. IV]. At time $t=0$, initial states $|\psi(0)\rangle=$ $|\psi\rangle$ are randomly sampled according to (15) or (37), and then evolve in time as $|\psi(t)\rangle=U_{t}|\psi\rangle$ with propagator $U_{t}=\exp \{-i H t / \hbar\}$, see also above Eq. (13). Finally, we recall that the dynamical typicality property (17) will be fulfilled in very good approximation for most initial states $|\psi\rangle$, provided $\rho$ in (16) satisfies (10), or equivalently (26).

Following Refs. [13, 26], we say that the system exhibits equilibration if the expectation value $\langle\psi(t)|O| \psi(t)\rangle$ remains very close to a constant value for the vast majority of all sufficiently large times $t$, i.e., after initial transients (relaxation processes) have died out. (Note that a small fraction of exceptional times $t$ is unavoidable due to quantum revival effects.) If this constant long-time value is furthermore (approximately) equal to the pertinent microcanonical expectation value, as predicted by textbook statistical mechanics, then we say that the sys- 
tem exhibits thermalization [13, 26]. In the following, we restrict ourselves to the question of equilibration, while the issue of thermalization is not pursued any further.

According to (17), most initial states $|\psi\rangle$ will exhibit equilibration if the mixed state $\rho(t)$ exhibits equilibration. The latter has been demonstrated under quite weak conditions on the spectrum of $H$ and on the initial state $\rho(0)=\rho$ in Refs. [13, 26]: Essentially, it is sufficient that the energy differences $E_{m}-E_{n}$ do not coincide for too many index pairs with $m \neq n$, which is the case for any generic Hamiltonian $H$ [13, 26], and that all energy levels are weakly populated, i.e.,

$$
\max _{n}\left\langle\chi_{n}|\rho| \chi_{n}\right\rangle \ll 1
$$

Observing that the left hand side of (66) is upper bounded by the maximal eigenvalue of $\rho$ in (19), which in turn is given by $p_{\max }$ in (25), one readily sees that our previously established precondition for dynamical typicality from (26) is at the same time sufficient to guarantee equilibration.

In summary, if the system under consideration is isolated, exhibits a generic energy spectrum, and exhibits dynamical typicality, then most initial states must also exhibit equilibration.

\section{PRACTICAL CONSIDERATIONS}

To explicitly determine the density operator $\rho$ in (43) and the concomitant $R$ in (21) by solving (35) for a given set of $\alpha_{k}$ 's may be quite cumbersome in practice (e.g., by numerical means). In fact, already in order to define the pertinent energy shell in Sec. VIIIA. one usually cannot avoid to diagonalize the Hamiltonian.

A radically different approach is to start out from some "reasonable ansatz" for $R$ in accordance with (21) and (43). In principle, one could then determine the corresponding $\alpha_{k}$ values in (35) a posteriori, but in practice some other way of justifying the proposed ansatz may even be more appropriate. Also the need to work with an energy shell may be circumvented due to the following observation: The actually relevant relations - for instance (11)-(27), (35), and (43) - remain well-defined even when working with the full Hilbert space of the considered model (see also Sec. VI), or, formally speaking, with the "largest possible energy shell" (its dimension may in principle be infinite, but numerically it will still be finite from the outset). Yet, a relatively "sharp" energy of the (typical) initial states $|\psi\rangle$ may be achieved, for instance, by setting $A_{1}=H$ and $A_{2}=H^{2}$ in (35) and choosing some suitable values of $\alpha_{1}$ and $\alpha_{2}$. Within our present approach, this amounts to evaluating the quantities

$$
\begin{aligned}
\bar{H} & :=\operatorname{Tr}\{\rho H\}, \\
\delta \bar{H}^{2} & :=\operatorname{Tr}\left\{\rho(H-\bar{H})^{2}\right\}=\operatorname{Tr}\left\{\rho H^{2}\right\}-\bar{H}^{2}
\end{aligned}
$$

by means of the proposed ansatz for $\rho$ and then to decide, whether such an energy distribution ("washed out energy window") is still acceptable to describe the problem at hand (note the similarities and differences compared to (41) and (5)).

In order to guarantee dynamical typicality properties, also (10) must be verified by the proposed ansatz $\rho$. As a consequence, instead of evaluating (35), (67), and (68) by means of $\rho$, the corresponding values of $\alpha_{k}, \bar{H}$, and $\delta \bar{H}^{2}$, which this ansatz entails, can also be approximated very well by means of a single (supposedly typical) pure state $|\psi\rangle$.

A simple example (see also Ref. [27]) is to choose $R$ in (21) proportional to $\exp \left\{-(H-U)^{2} / 4 \sigma^{2}\right\}$ and hence $\rho$ in (19) as

$$
\begin{aligned}
\rho & =\frac{1}{Y} \exp \left\{-\frac{(H-U)^{2}}{2 \sigma^{2}}\right\}=\sum_{n=1}^{N} p_{n}|n\rangle\langle n|, \\
p_{n} & :=\frac{1}{Y} \exp \left\{-\frac{\left(E_{n}-U\right)^{2}}{2 \sigma^{2}}\right\}
\end{aligned}
$$

where $U$ and $\sigma$ are real parameters, $Y$ is a normalization constant, $E_{n}$ and $|n\rangle$ are the eigenvalues and eigenvectors of $H$, and (22) has been exploited in (69). Assuming that $\sigma$ is much larger than the typical distance between neighboring energy levels $E_{n}$, and that the mean level spacing can be approximated by a constant value $\Delta$ within a neighborhood of $U$ extending over several $\sigma$ 's, sums over $n$ can be readily approximated by integrals in (67)- (669), yielding

$$
\begin{aligned}
\bar{H} & \simeq U, \\
\delta \bar{H} & \simeq \sigma \\
Y & \simeq \sqrt{2 \pi} \sigma / \Delta .
\end{aligned}
$$

With (25) and (70) we can conclude that that $p_{\max } \leq$ $1 / Y$. Since we assumed $\sigma \gg \Delta$ it follows that our ansatz (69) satisfies (26) and hence (10). Moreover, we see that the location and width of the "washed out energy window" in (67), (68) can be controlled via the parameters $U$ and $\sigma$ in (69).

According to (39), normalized vectors $|\psi\rangle$ with equal values of $\left\langle\psi\left|\rho^{-1}\right| \psi\right\rangle=\sum_{n=1}^{N}|\langle\psi \mid n\rangle|^{2} / p_{n}$ are realized with equal probability. Moreover, by means of (22), our ansatz (69) can be rewritten in the form (43) with $K=\infty$ and

$$
\begin{aligned}
\lambda_{k} & :=Y \text { for } k=0,1,2, \ldots, \\
A_{k} & :=\frac{1}{\left(2 \sigma^{2}\right)^{k} k !}(H-U)^{2 k} \text { for } k=1,2,3, \ldots .
\end{aligned}
$$

Under the same assumption as above (71) it follows with (35) that

$$
\alpha_{k} \simeq(2 k) ! /\left(2^{k} k !\right)^{2} \simeq 1 / \sqrt{\pi k},
$$

where the last relation is obtained for large $k$ by Stirling's approximation. In passing we note that $K \ll N$ has been assumed in Sec. VIII for the sole reason that otherwise (35) may not be solvable for almost every a priori fixed 
set of $\alpha_{k}$ 's. In contrast, here $\rho$ is "given" and the corresponding $\alpha_{k}$ 's are determined via (35) a posteriori, so that $K \rightarrow \infty$ does not give rise to any problems.

From the physical viewpoint, (69) represents yet another equilibrium ensemble of the type discussed in Sec. VII It is therefore plausible that the concomitant expectation values and hence the typicality properties for this equilibrium ensemble will be equivalent (practically equal) to those of, e.g., the microcanonical ensemble from (32) or the canonical ensemble from (33). However, from the mathematical viewpoint this equivalence of the ensembles is far from being obvious and in fact is strictly speaking even untenable for arbitrary observables. Rather, the equivalence is only expected to be true for observables which satisfy the so-called eigenstate thermalization hypothesis (ETH) 28 33].

It is straightforward to formally generalized (69) for non-equilibrium situations, for instance by choosing [34, 35 .

$$
\begin{aligned}
\rho & =Y^{-1} \exp \{-S\} \\
S & :=\frac{(H-U)^{2}}{2 \sigma^{2}}+\frac{(G-V)^{2}}{2 \tau^{2}},
\end{aligned}
$$

where $U, V, \sigma, \tau \in \mathbb{R}$ are parameters and $G$ is some observable, by which the initial disequilibrium can be characterized, and which in general does not commute with $H$. As before, such an ansatz is again of the general form (43) with (74) and $A_{k}:=S^{k} / k$ ! for $k=1,2,3, \ldots$, while the corresponding $\alpha_{k}$ values can be readily determined a posteriori via (35). Likewise, (10) will be satisfied whenever $Y \gg 1$. It is again reasonable to expect that such an ansatz may effectively generate some "washed out energy window" and simultaneously some reasonably sharply defined non-equilibrium initial conditions for the observable $G$. The actual energy window follows again by evaluating (67) and (68), and similarly for the initial distribution of measurement outcomes for $G$. But unlike in the equilibrium case, such simple relations between the pertinent eigenvalues and eigenvectors as in (69), (70) are no longer available. As a consequence, also (71) and (72) will in general no longer be fulfilled. However, for a reasonable choice of parameters ( $\tau$ not too small, $V$ not too far away from the equilibrium expectation value of $G$ ) it appears plausible that the implication of (73), namely that $\rho$ complies with (10), remains valid, c.f. below. In this case typicality is expected, i.e., most pure states of the ensembles as given in (1) or (15) will reliably mimic the behavior of $\rho$. Other than that, and most importantly, it is now much more difficult to say which actual (e.g. experimental or numerical) situation and which observables can be faithfully approximated in a quantitative way within our present typicality approach by means of such an ansatz for $\rho$.

On the other hand, the numerical advantage of such an approach is quite remarkable: Similarly as described at the end of Sec. VII, the operator $R$ in (1) can be rewritten due to (21) and (77) as $\gamma \exp \{-S / 2\}$ with $\gamma=Y^{-1 / 2}$. Setting temporarily $\gamma=1$, the evaluation of $|\phi\rangle$ in (1) is possible by means of numerically very efficient imaginary time propagation techniques [34 37], yielding the same normalized random vector $|\psi\rangle$ in (15) as one would have obtained for $\gamma=Y^{-1 / 2}$. But by working with $\gamma=1$, the resulting random number $\langle\phi \mid \phi\rangle$ will be typically very close to $Y$. In other words, the same numerically obtained random vector $|\phi\rangle$ can be used not only to deduce the usual dynamical typicality properties but also to check whether the above derived sufficient condition $Y \gg 1$ for (10) is fulfilled.

\section{SUMMARY AND CONCLUSION}

We examined ensembles of pure states which are distributed in a high-dimensional Hilbert space such that states featuring equal expectation values for a set of $K$ observables $A_{k}$ occur with equal probability. These ensembles are usually not Haar-measure invariant. Rather they may be considered as unbiased ensembles, given an a priori knowledge about the expectation values of the above observables $A_{k}$. Such an a priori knowledge is characteristic of the initial situation of non-equilibrium experiments in which the relaxation of some observables is monitored. Under rather mild conditions, these ensembles are found to exhibit strong typicality properties, i.e., the overwhelming majority of individual states from these ensembles feature very similar expectation values for a largely arbitrary observable at the initial as well as at any later instance of time. In particular, this observable may be different from the above $A_{k}$ 's or linear combinations thereof.

In other words, we demonstrated that knowing the initial expectation values of a few observables is sufficient to produce a very reliable guess about the time-dependent expectation values of another, possibly very different observable. In particular this gives rise to so-called " $\mathrm{dy}$ namical typicality": Knowledge of some initial expectation value $\langle\psi|O(0)| \psi\rangle$ suffices to produce a very reliable guess on the expectation value of the same observable at any later time $\langle\psi|O(t)| \psi\rangle$.

We discussed in detail three ensembles which exhibit the above "unbiasedness" but differ in the actual probability with which pure states featuring some common expectation values actually occur in the ensemble. This leads to mathematically well-defined qualitative differences between these ensembles. It is demonstrated, however, that the above guess on the expectation value of some further observable only varies very slightly with the choice of a specific ensemble. One of the discussed ensembles may be efficiently numerically generated on a computer. This allows for the construction of a numerical tool with which the behavior of ensembles, mixed states, etc. may be mimicked by a single, properly chosen pure state. 


\section{Acknowledgments}

Stimulating discussions with Ben N. Balz and Lennart Dabelow are greatefully acknowledged. We also thank Shelly Goldstein for explaining the derivation of Eq. (18a) in 24]. This work was supported by the Deutsche Forschungsgemeinschaft (DFG) within the Research Unit FOR 2692 under Grants No. 397303734 and 397107022.

\section{Appendix A: Time-dependent Hamiltonians}

As mentioned above (13), the system dynamics may be generated in full generality by an explicitly timedependent Hamiltonian. In case that the two Hilbert spaces $\mathcal{H}$ and $\mathcal{H}^{\prime}$ disagree (see Sec. VI), certain details of the so far formalism require some refinements:

In the case of an explicitly time dependent Hamiltonian $H^{\prime}(t)$, we still can conclude from Sec. VI that $H(0):=P^{\prime} H^{\prime}(0) P^{\prime}=H^{\prime}(0)$. Hence, the discussion in Sec. III still remains valid as long as only the time $t=0$ matters. The first relevant modification thus concerns the propagator $U_{t}^{\prime}$ (see above (13)), which is a priori an operator on $\mathcal{H}^{\prime}$. For time independent Hamiltonians, one sees that the relation $U_{t}:=P^{\prime} U_{t}^{\prime} P^{\prime}=U_{t}^{\prime}$ holds for all $t$, hence the transition from $\mathcal{H}^{\prime}$ to $\mathcal{H}$ is trivial. The same no longer applies for time-dependent Hamiltonians. Yet, (13) remains valid if we define $O_{t}:=P^{\prime} O_{t}^{\prime} P^{\prime}$, and replace (14) by

$$
O_{t}^{\prime}:=\left(U_{t}^{\prime}\right)^{\dagger} O^{\prime} U_{t}^{\prime}
$$

Analogously, $\rho^{\prime}(t):=\left(U_{t}^{\prime}\right)^{\dagger} \rho^{\prime}(0)^{\prime} U_{t}^{\prime}$ is now an operator on $\mathcal{H}^{\prime}$ and (17) has to be replaced by

$$
\langle\psi(t)|O| \psi(t)\rangle=\operatorname{Tr}^{\prime}\left\{\rho^{\prime}(t) O^{\prime}\right\},
$$

where $\operatorname{Tr}^{\prime}$ indicates the trace in $\mathcal{H}^{\prime}$. Note that (A2) and (17) are still equivalent for $t=0$.

In spite of these modifications, all the dynamical typicality properties discussed below (17) and in Sec. VI still remain valid even for explicitly time-dependent Hamiltonians.

\section{Appendix B: Derivation of Equation (39)}

In this Appendix we show that the probability distribution of the random vectors in (37) is given by (39).

As said below (11), the real and imaginary parts of the complex numbers $c_{n}$ are given by independent, Gaussian distributed random variables of mean zero and variance $1 / 2$. Accordingly, the $N$-dimensional complex vectors

$$
\mathbf{c}:=\left(c_{1}, \ldots, c_{N}\right)
$$

can and will be interpreted from now on as $2 N$ dimensional real vectors, whose probability distribution is given by

$$
\begin{aligned}
w_{1}(\mathbf{c}) & :=\pi^{-N} \exp \left\{-\|\mathbf{c}\|^{2}\right\}, \\
\|\mathbf{c}\| & :=\sqrt{\sum_{n=1}^{N}\left|c_{n}\right|^{2}} .
\end{aligned}
$$

Viewing (38) as a transformation of random variables, the connection between the corresponding probability distributions $w_{1}(\mathbf{c})$ and $w(\mathbf{y})$ is given according to textbook probability theory by

$$
\begin{aligned}
w(\mathbf{y}) & :=\int d \mathbf{c} w_{1}(\mathbf{c}) \delta(\mathbf{y}-\tilde{\mathbf{y}}(\mathbf{c})), \\
\tilde{y}_{n}(\mathbf{c}) & :=\frac{\sqrt{p_{n}} c_{n}}{\sqrt{\sum_{n=1}^{N} p_{n}\left|c_{n}\right|^{2}}}
\end{aligned}
$$

with the following definitions for any $\mathbf{z} \in \mathbb{C}^{N}$ :

$$
\begin{aligned}
d \mathbf{z} & :=\prod_{n=1}^{N} d\left(\operatorname{Re} z_{n}\right) d\left(\operatorname{Im} z_{n}\right), \\
\delta(\mathbf{z}) & :=\prod_{n=1}^{N} \delta\left(\operatorname{Re} z_{n}\right) \delta\left(\operatorname{Im} z_{n}\right) .
\end{aligned}
$$

Going over from the integration variables $c_{n}$ to $z_{n}:=$ $\sqrt{p_{n}} c_{n}$ yields

$$
\begin{aligned}
w(\mathbf{y}) & =\int d \mathbf{z} w_{2}(\mathbf{z}) \delta(\mathbf{y}-\mathbf{z} /\|\mathbf{z}\|), \\
w_{2}(\mathbf{z}) & :=\frac{1}{\pi^{N} \operatorname{det}(\rho)} \exp \left\{-\sum_{n=1}^{N} \frac{\left|z_{n}\right|^{2}}{p_{n}}\right\},
\end{aligned}
$$

where $\operatorname{det}(\rho):=\prod_{n=1}^{N} p_{n}$ is the determinant of $\rho$ from (19). Multiplying the right hand side of (B8) by $1=$ $\int_{0}^{\infty} d r \delta(\|\mathbf{z}\|-r)$ result in

$$
w(\mathbf{y})=\int_{0}^{\infty} d r \int d \mathbf{z} w_{2}(\mathbf{z}) \delta(\mathbf{y}-\mathbf{z} / r) \delta(\|\mathbf{z}\|-r) .
$$

Going over from the integration variables $z_{n}$ to $v_{n}:=$ $z_{n} / r$ implies

$$
\begin{aligned}
w(\mathbf{y}) & =\int_{0}^{\infty} d r \int d \mathbf{v} r^{2 N} w_{2}(r \mathbf{v}) \delta(\mathbf{y}-\mathbf{v}) \delta(r\|\mathbf{v}\|-r) \\
& =\int_{0}^{\infty} d r r^{2 N} w_{2}(r \mathbf{y}) \delta(r(\|\mathbf{y}\|-1)) . \quad(\mathrm{B} 11)
\end{aligned}
$$

Defining the Heaviside step function for any $x \in \mathbb{R}$ as $\Theta(x):=\int_{-\infty}^{x} d y \delta(y)$, it follows that $\Theta^{\prime}(x)=\delta(x)$ and $\Theta(r x)=\Theta(x)$ for any $r>0$. One thus can conclude that $\delta(x)=d \Theta(x) / d x=d \Theta(r x) / d x=r \Theta^{\prime}(r x)=r \delta(r x)$ and therefore $\delta(r x)=\delta(x) / r$. Exploiting the latter identity and (B9), we can rewrite (B11) as

$$
\begin{aligned}
w(\mathbf{y}) & =\frac{\delta(\|\mathbf{y}\|-1)}{\pi^{N} \operatorname{det}(\rho)} \int_{0}^{\infty} d r r^{2 N-1} e^{-r^{2} g(\mathbf{y})}, \\
g(\mathbf{y}) & :=\sum_{n=1}^{N} \frac{\left|y_{n}\right|^{2}}{p_{n}}
\end{aligned}
$$


Going over from the integration variable $r$ to $t:=g(\mathbf{y}) r^{2}$ and exploiting the common properties of the gamma function, one finds that

$$
w(\mathbf{y})=\frac{\delta(\|\mathbf{y}\|-1)}{\pi^{N} \operatorname{det}(\rho)} \frac{(N-1) !}{2 g(\mathbf{y})^{N}} .
$$

Next we observe that (37) represents a one-to-one correspondence between any possible vector $|\psi\rangle \in \mathcal{H}$ and any possible vector $\mathbf{y}$ in $\mathbb{C}^{N}$, or equivalently in $\mathbb{R}^{2 N}$. Accordingly, and as said already below (37), the probability distribution $w(|\psi\rangle)$ of the vectors $|\psi\rangle \in \mathcal{H}$ is by definition given by the probability distribution of the corresponding random numbers $y_{n}$ in (37), i.e., by $w(\mathbf{y})$ from (B14). Moreover, $\|\mathbf{y}\|$ can be readily identified with $\|\psi\|$. Likewise, one can rewrite (B13) by means of (23) and (37) as

$$
g(\mathbf{y})=\left\langle\psi\left|\rho^{-1}\right| \psi\right\rangle
$$

Taking all this onto account in (B14), one finally recovers (39).

\section{Appendix C: Derivation of Equation (43)}

In this Appendix we show that if there exists as function $h: \mathbb{R}^{K} \rightarrow \mathbb{R}$ which fulfills (41) for all normalized vectors $|\psi\rangle \in \mathcal{H}$, then $\rho$ must be of the form Eq. (433) (under the tacit extra assumption that $h$ is twice continuously differentiable).

Clearly, (41) is satisfied for all normalized vectors $|\psi\rangle \in$ $\mathcal{H}$ if and only if

$$
\left\langle\phi\left|\rho^{-1}\right| \phi\right\rangle=\|\phi\|^{2} h\left(a_{\phi}^{1}, \ldots, a_{\phi}^{K}\right)
$$

with $a_{\phi}^{k}:=\left\langle\phi\left|A_{k}\right| \phi\right\rangle /\|\phi\|^{2}$ and $\|\phi\|:=\langle\phi \mid \phi\rangle^{1 / 2}$ is satisfied for all vectors $|\phi\rangle \in \mathcal{H}$ of non-zero but otherwise arbitrary norm.

Rewriting (23) by means of the abbreviation $q_{n}:=$ $1 / p_{n}$ as

$$
\rho^{-1}=\sum_{n=1}^{N} q_{n}|n\rangle\langle n|
$$

we now focus in (C1) on the special choice

$$
|\phi\rangle=|m\rangle+\sqrt{\epsilon} z|n\rangle
$$

for arbitrary $m \neq n, z \in \mathbb{C}$, and $\epsilon \in \mathbb{R}_{0}^{+}$(later we will set $\epsilon=1$ and restrict ourselves to small $|z|)$. Introducing (C2) and (C3) into (C1) yields

$$
\begin{aligned}
& q_{m}+\epsilon z^{2} q_{n}=\left(1+\epsilon z^{2}\right) h\left(x_{1}, \ldots, x_{K}\right), \\
& x_{k}:=\frac{A_{m m}^{k}+\sqrt{\epsilon} Z_{m n}^{k}+\epsilon z^{2} A_{n n}^{k}}{1+\epsilon z^{2}} \\
& A_{m n}^{k}:=\left\langle m\left|A_{k}\right| n\right\rangle \\
& Z_{m n}^{k}:=z A_{m n}^{k}+\left(z A_{m n}^{k}\right)^{*}=2 \operatorname{Re}\left(z A_{m n}^{k}\right) .
\end{aligned}
$$

Differentiating (C4) with respect to $\epsilon$ and then setting $\epsilon=1$ yields

$$
\begin{aligned}
& z^{2} q_{n}-z^{2} h\left(x_{1}, \ldots, x_{K}\right)=\sum_{k=1}^{K} y_{k} \frac{\partial h\left(x_{1}, \ldots, x_{K}\right)}{\partial x_{k}}, \\
& y_{k}:=\frac{Z_{m n}^{k}}{2}+z^{2}\left(A_{n n}-\frac{A_{m m}^{k}+Z_{m n}^{k}+z^{2} A_{n n}^{k}}{1+z^{2}}\right) .
\end{aligned}
$$

Upon eliminating $h\left(x_{1}, \ldots, x_{K}\right)$ in (C8) by means of (C4) (with $\epsilon=1$ ) one obtains

$$
\frac{z^{2}}{1+z^{2}}\left(q_{n}-q_{m}\right)=\sum_{k=1}^{K} y_{k} \frac{\partial h\left(x_{1}, \ldots, x_{K}\right)}{\partial x_{k}} .
$$

Focusing on small $|z|$ and observing that $Z_{m n}^{k}$ in (C7) is of first order in $z$, it follows from (C5) and (C9) that

$$
\begin{gathered}
y_{k} \frac{\partial h\left(x_{1}, \ldots, x_{K}\right)}{\partial x_{k}}=\left(\frac{Z_{m n}^{k}}{2}+z^{2}\left(A_{n n}^{k}-A_{m m}^{k}\right)\right) h_{k}^{m} \\
+\frac{1}{2} \sum_{j=1}^{K} Z_{m n}^{j} Z_{m n}^{k} h_{j k}^{m}+\mathcal{O}\left(z^{3}\right), \quad(\mathrm{C} 11) \\
h_{k}^{m}:=\frac{\partial h\left(A_{m m}^{1}, \ldots, A_{m m}^{K}\right)}{\partial x_{k}}, \\
h_{j k}^{m}:=\frac{\partial^{2} h\left(A_{m m}^{1}, \ldots, A_{m m}^{K}\right)}{\partial x_{j} \partial x_{k}} .
\end{gathered}
$$

Since $h$ is a real valued function, also its derivatives in (C12) and (C13) are real numbers.

By collecting terms of first order in $z$ one can infer from (C10) and (C11) that

$$
\begin{aligned}
& 0=\sum_{k=1}^{K} Z_{m n}^{k} h_{k}^{m}=z G_{m n}^{m}+\left(z G_{m n}^{m}\right)^{*}, \\
& G_{m n}^{l}:=\sum_{k=1}^{K} A_{m n}^{k} h_{k}^{l},
\end{aligned}
$$

where we exploited (C7) on the right hand side of (C14). Since $z \in \mathbb{C}$ in (C14) is still arbitrary, it follows that

$$
G_{m n}^{m}=0
$$

where, as said below (C3), the indices $m$ and $n$ must be different but otherwise may be arbitrary.

Analogously, by collecting terms of second order in $z$ one can infer from (C10) and (C11) that

$z^{2}\left(q_{n}-q_{m}\right)=z^{2}\left(G_{n n}^{m}-G_{m m}^{m}\right)+\sum_{j, k=1}^{K} \frac{Z_{m n}^{j} Z_{m n}^{k} h_{j k}^{m}}{2}$

One readily confirms that apart from $z$, all other terms in (C17) are real numbers. Since $z \in \mathbb{C}$ in (C17) is still arbitrary, one can conclude that

$$
q_{n}-q_{m}=G_{n n}^{m}-G_{m m}^{m}
$$


for arbitrary $m \neq n$. Obviously, this result also remains valid for $m=n$.

Considering $m$ as arbitrary but fixed and $n$ as variable, we can rewrite (C18) with (C15) as

$$
\begin{aligned}
q_{n} & =\lambda_{0}^{m}+\sum_{k=1}^{K} \lambda_{k}^{m} A_{n n}^{k}, \\
\lambda_{0}^{m} & :=q_{m}-G_{m m}^{m}, \\
\lambda_{k}^{m} & :=h_{k}^{m} .
\end{aligned}
$$

Note that all quantities in (C20) are real numbers. Considering $m$ as arbitrary but fixed, C19 amounts to a linear relation between the $q_{n}$ and the $A_{n n}^{k}$ for all $n=1, \ldots, N$. If we choose some different value $m$ as our fixed index, the same relation must again be true for all $n=1, \ldots, N$. Since the left hand side of (C19) is independent of $m$ and since $K \leq N$ (see main text), this is only possible if the coefficients $\lambda_{0}^{m}, \ldots, \lambda_{K}^{m}$ are actually the same for any choice of $m$. In other words, the coefficients are independent of $m$ and hence their upper index $m$ can be omitted. Accordingly, (C19) takes the form

$$
q_{n}=\lambda_{0}+\sum_{k=1}^{K} \lambda_{k} A_{n n}^{k}
$$

for all $n=1, \ldots, N$. Likewise, C15 can be rewritten with (C21) as

$$
G_{m n}^{l}=\sum_{k=1}^{K} \lambda_{k} A_{m n}^{k}
$$

independent of $l$. Finally, (C16) thus amounts to

$$
\sum_{k=1}^{K} \lambda_{k} A_{m n}^{k}=0
$$

for all $m \neq n$.

Taking into account (C2), (C6), (C22), and (C24), one readily confirms that

$$
\left\langle m\left|\rho^{-1}\right| n\right\rangle=\lambda_{0} \delta_{m n}+\sum_{k=1}^{K} \lambda_{k}\left\langle m\left|A_{k}\right| n\right\rangle
$$

for all $m, n$. Consequently,

$$
\rho^{-1}:=\lambda_{0} \mathbb{1}+\sum_{k=1}^{K} \lambda_{k} A_{k}
$$

and thus Eq. (43) from the main text follows.

Introducing (43) into (41) implies that

$$
h\left(v_{1}, \ldots, v_{K}\right)=\lambda_{0}+\sum_{k=1}^{K} \lambda_{k} v_{k} .
$$

In turn, it is straightforward to verify that this function with arbitrary real coefficients $\lambda_{0}, \ldots, \lambda_{K}$ fulfills all the equations and properties which we encountered in the course of the above calculations. Our considerations also show that only functions $h$ of the form (C27) satisfy all those equations and properties.

\section{Appendix D: Reducing the number of equations}

In view of (43) it follows that

$$
\operatorname{Tr}\left\{\rho\left[\lambda_{0} \mathbb{1}+\sum_{k=1}^{K} \lambda_{k} A_{k}\right]\right\}=N
$$

and hence

$$
\lambda_{0} \operatorname{Tr}\{\rho\}+\sum_{k=1}^{K} \lambda_{k} \operatorname{Tr}\left\{\rho A_{k}\right\}=N .
$$

Together with conditions (3) and (35) we can conclude that

$$
\lambda_{0}=N-\sum_{k=1}^{K} \lambda_{k} \alpha_{k}
$$

In other words, among the $K+1$ conditions (3) and (35) we can choose an arbitrary one and replace it by (D3).

Defining $y_{k}:=-\lambda_{k} / N$ and introducing (D3) into (43) yields

$$
\rho=\frac{1}{N}\left[\mathbb{1}-\sum_{k=1}^{K} y_{k}\left(A_{k}-\alpha_{k}\right)\right]^{-1}
$$

where the $K$ parameters $y_{k}$ are fixed by the $K+1$ conditions (3) and (35), among which one will be automatically fulfilled if all the $K$ others are fulfilled. Moreover, the $y_{k}$ 's must be so that (D4) is a strictly positive definite operator (cf. (44)).

A more detailed exploration of the special case $K=1$ has been carried out in [12]. In particular, such a closer inspection shows that the minus sign in the above definition of the $y_{k}$ is convenient and natural.
[1] A. Engel and C. Van den Broeck, Statistical Mechanics of Learning (Cambridge University Press, 2001)

[2] S. Goldstein, J. L. Lebowitz, R. Tumulka, and N. Zanghi, Phys. Rev. Lett. 96, 050403 (2006)

[3] S. Popescu, A. J. Short, and A. Winter, Nature Phys. 2,
754 (2006)

[4] S. Lloyd, Ph.D. Thesis, The Rockefeller University (1988), Chapter 3, arXiv:1307.0378

[5] C. Bartsch and J. Gemmer, Phys. Rev. Lett. 102, 110403 (2009) 
[6] A. Sugita, Nonlinear Phenom. Complex Syst. 10, 192-195 (2007)

[7] P. Reimann, Phys. Rev. Lett. 99, 160404 (2007)

[8] J. Gemmer, M. Michel, and G. Mahler, Quantum Thermodynamics (2nd edition, Springer, Berlin, Heidelberg, 2009)

[9] S. Sugiura and A. Shimizu, Phys. Rev. Lett. 108, 240401 (2012)

[10] H. Tasaki, J. Stat. Phys. 163, 937 (2016)

[11] B. N. Balz, J. Richter, J. Gemmer, R. Steinigeweg, and P. Reimann, Dynamical typicality for initial states with a preset measurement statistics of several commuting observables, in Thermodynamics in the Quantum Regime; Fundamental Aspects and New Directions, edited by F. Binder, L. A. Correa, C. Gogolin, J. Anders, and G. Adesso, Springer, Berlin, Heidelberg, 2018.

[12] P. Reimann, Phys. Rev. E 97, 062129 (2018)

[13] N. Linden, S. Popescu, A. J. Short, and A. Winter, Phys. Rev. E 79, 061103 (2009)

[14] A. S. L. Malabarba, L. P. Garcia-Pintos, N. Linden, T. C. Farrelly, and A. J. Short, Phys. Rev. E 90, 012121 (2014)

[15] P. Reimann, Phys. Rev. Lett. 115, 010403 (2015)

[16] C. Bartsch and J. Gemmer, Europhys. Lett. 118, 10006 (2017)

[17] S. Sugiura and A. Shimizu, Phys. Rev. Lett. 111, 010401 (2013)

[18] A. Hams and H. De Raedt, Phys. Rev. E 62, 4365 (2000)

[19] R. Alben, M. Blume, H. Krakauer, and L. Schwartz, Phys. Rev B 12, 4090 (1975); P. de Vries and H. De Raedt, Phys. Rev. B 47, 7929 (1993); T. Iitaka, S. Nomura, H. Hirayama, X. Zhao, Y. Aoyagi, and T. Sugano, Phys. Rev. E 56, 1222 (1997)

[20] M. Hyuga, S. Sugiura, K. Sakai, and A. Shimizu, Phys. Rev. B 90, 121110(R) (2014)
[21] P. Reimann and J. Gemmer, Phys. Rev. E 99, 012125 (2019)

[22] B. V. Fine, Phys. Rev. E 80, 051130 (2009)

[23] M. P. Müller, D. Gross, and J. Eisert, Commun. Math. Phys. 303, 785 (2011)

[24] S. Goldstein, J. L. Lebowitz, R. Tumulka, and N. Zanghi, J. Stat. Phys. 125, 1197 (2006)

[25] P. Reimann, J. Stat. Phys. 132, 921 (2008)

[26] P. Reimann, Phys. Rev. Lett. 101, 190403 (2008); A. J. Short, New J. Phys. 13, 053009 (2011); P. Reimann and M. Kastner, New J. Phys. 14, 043020 (2012); A. J. Short and T. C. Farrelly, New J. Phys. 14, 013063 (2012); B. N. Balz and P. Reimann, Phys Rev. E 93, 062107 (2016)

[27] R. Steinigeweg, A. Khodja, H. Niemeyer, C. Gogolin, and J. Gemmer, Phys. Rev. Lett. 112, 130403 (2014)

[28] J. M. Deutsch, Phys. Rev. A 43, 2046 (1991)

[29] M. Srednicki, Phys. Rev. E 50, 888 (1994)

[30] M. Srednicki, J. Phys. A 29, L75 (1996)

[31] M. Rigol, V. Dunjko, and M. Olshanii, Nature (London) 452, 854 (2008)

[32] L. D'Alessio, Y. Kafri, A. Polkovnikov, and M. Rigol, Adv. Phys. 65, 239 (2016)

[33] C. Gogolin and J. Eisert, Rep. Prog. Phys. 79, 056001 (2016)

[34] A. Khodja, R. Steinigeweg, and J. Gemmer, Phys. Rev. E 91, 012120 (2015)

[35] A. Khodja, D. Schmidtke, and J. Gemmer, Phys. Rev. E 93, $042101(2016)$

[36] D. Schmidtke, L. Knipschild, M. Campisi, R. Steinigeweg, and J. Gemmer, Phys. Rev. E 98, 012123 (2018)

[37] F. Jin, R. Steinigeweg, H. De Raedt, K. Michielsen, M. Campisi, and J. Gemmer, Phys. Rev. E, 94, 012125, (2016) 